\title{
Wind TURBINE AND TURBOMACHINERY \\ Computational Analysis With the ALE AND Space-Time Variational Multiscale Methods AND ISOGEOMETRIC DISCRETIZATION
}

\author{
Yuri BAZILEVS ${ }^{1, *}$, Kenji TAKIZAWA ${ }^{2}$, Tayfun E. TEZDUYAR ${ }^{2,3}$, \\ Ming-Chen HSU ${ }^{4}$, Yuto OTOGURO ${ }^{2}$, Hiroki MOCHIZUKI $I^{2}$, Michael C.H. WU ${ }^{1}$
}

${ }^{1}$ Brown University, Providence, Rhode Island, USA

${ }^{2}$ Waseda University, Tokyo, Japan

${ }^{3}$ Rice University, Houston, Texas, USA

${ }^{4}$ Iowa State University, Ames, Iowa, USA

*Corresponding Author: Yuri BAZILEVS (email: yuri_bazilevs@brown.wdu)

(Received: 12-Feb-2020; accepted: 14-Feb-2020; published: 31-Mar-2020)

DOI: http://dx.doi.org/10.25073/jaec.202041.278

\begin{abstract}
The challenges encountered in computational analysis of wind turbines and turbomachinery include turbulent rotational flows, complex geometries, moving boundaries and interfaces, such as the rotor motion, and the fluid-structure interaction (FSI), such as the FSI between the wind turbine blade and the air. The Arbitrary Lagrangian-Eulerian (ALE) and Space-Time (ST) Variational Multiscale (VMS) methods and isogeometric discretization have been effective in addressing these challenges. The ALE-VMS and ST-VMS serve as core computational methods. They are supplemented with special methods like the Slip Interface (SI) method and ST Isogeometric Analysis with NURBS basis functions in time. We describe the core and special methods and present, as examples of challenging computations performed, computational analysis of horizontaland vertical-axis wind turbines and flow-driven string dynamics in pumps.
\end{abstract}

\section{Keywords}

Wind turbine, pump, string dynamics, FSI, space-time VMS method, ALE-VMS method, isogeometric analysis.

\section{Introduction}

Complexity level and reliability of computational analysis of wind turbines and turbomachinery define the practical value of the computations. The Arbitrary Lagrangian-Eulerian (ALE) and Space-Time (ST) Variational Multiscale (VMS) methods and isogeometric discretization are now enabling in wind turbine and turbomachinery computational analysis a complexity level that reflects the actual conditions, with reliable results (see, for example, [1-4]). The computational challenges encountered in this class of problems include turbulent rotational flows, complex geometries, moving boundaries and interfaces, such as the rotor motion, and the fluid--structure interaction (FSI), such as the FSI between the wind turbine blade and the air. 
Our core methods in addressing the computational challenges are the ALE-VMS [5] and ST-VMS [6]. We have a number special methods used in combination with them. The special methods used in combination with the ST-VMS include the ST Slip Interface (ST-SI) method [1, 7], ST Isogeometric Analysis (ST-IGA) [6, 8, 9], ST/NURBS Mesh Update Method (STNMUM) [8], a general-purpose NURBS mesh generation method for complex geometries [10,11], and a one-way-dependence model for the string dynamics [12]. The special methods used in combination with the ALE-VMS include weak enforcement of no-slip boundary conditions [13-15] and "sliding interfaces" $[16,17]$ (the acronym "SI" will also indicate that).

We will provide an overview of the core and special methods and present examples of challenging computations performed with these methods, including computational analysis of horizontal- and vertical-axis wind turbines (HAWTs and VAWTs) and flow-driven string dynamics in pumps. Much of the material presented in this review article has been extracted from [18] and the earlier articles written by the authors.

We provide the governing equations in Section 2. The core and special methods and other methods are described in Sections 3-10. In Sections 11 and 12, as examples of the ST computations, we present flow-driven string dynamics in a pump and aerodynamics of a VAWT. In Section 13, as an example of the ALE computations, we present FSI of a HAWT with rotor-tower coupling. The concluding remarks are given in Section 14 .

\section{Governing equations}

\subsection{Incompressible flow}

Let $\Omega_{t} \subset \mathbb{R}^{n_{\mathrm{sd}}}$ be the spatial domain with boundary $\Gamma_{t}$ at time $t \in(0, T)$, where $n_{\text {sd }}$ is the number of space dimensions. The subscript $t$ indicates the time-dependence of the domain. The Navier-Stokes equations of incompressible flows are written on $\Omega_{t}$ and $\forall t \in(0, T)$ as

$$
\begin{array}{r}
\rho\left(\frac{\partial \mathbf{u}}{\partial t}+\mathbf{u} \cdot \nabla \mathbf{u}-\mathbf{f}\right)-\nabla \cdot \boldsymbol{\sigma}=\mathbf{0}, \\
\boldsymbol{\nabla} \cdot \mathbf{u}=0,
\end{array}
$$

where $\rho, \mathbf{u}$ and $\mathbf{f}$ are the density, velocity and body force. The stress tensor $\boldsymbol{\sigma}(\mathbf{u}, p)=-p \mathbf{I}+$ $2 \mu \boldsymbol{\varepsilon}(\mathbf{u})$, where $p$ is the pressure, $\mathbf{I}$ is the identity tensor, $\mu=\rho \nu$ is the viscosity, $\nu$ is the kinematic viscosity, and the strain rate $\varepsilon(\mathbf{u})=$ $\left(\nabla \mathbf{u}+(\boldsymbol{\nabla u})^{T}\right) / 2$. The essential and natural boundary conditions for Eq. (1) are represented as $\mathbf{u}=\mathbf{g}$ on $\left(\Gamma_{t}\right)_{\mathbf{g}}$ and $\mathbf{n} \cdot \boldsymbol{\sigma}=\mathbf{h}$ on $\left(\Gamma_{t}\right)_{\mathbf{h}}$, where $\mathbf{n}$ is the unit normal vector and $\mathbf{g}$ and $\mathbf{h}$ are given functions. A divergence-free velocity field $\mathbf{u}_{0}(\mathbf{x})$ is specified as the initial condition.

\subsection{Structural mechanics}

In this article we will not provide any of our formulations requiring fluid and structure definitions simultaneously; we will instead give reference to earlier journal articles where the formulations were presented. Therefore, for notation simplicity, we will reuse many of the symbols used in the fluid mechanics equations to represent their counterparts in the structural mechanics equations. To begin with, $\Omega_{t} \subset \mathbb{R}^{n_{\text {sd }}}$ and $\Gamma_{t}$ will represent the structure domain and its boundary. The structural mechanics equations are then written, on $\Omega_{t}$ and $\forall t \in(0, T)$, as

$$
\rho\left(\frac{\mathrm{d}^{2} \mathbf{y}}{\mathrm{d} t^{2}}-\mathbf{f}\right)-\nabla \cdot \boldsymbol{\sigma}=\mathbf{0},
$$

where $\mathbf{y}$ and $\boldsymbol{\sigma}$ are the displacement and Cauchy stress tensor. The essential and natural boundary conditions for Eq. (3) are represented as $\mathbf{y}=$ $\mathbf{g}$ on $\left(\Gamma_{t}\right)_{\mathrm{g}}$ and $\mathbf{n} \cdot \boldsymbol{\sigma}=\mathbf{h}$ on $\left(\Gamma_{t}\right)_{\mathbf{h}}$. The Cauchy stress tensor can be obtained from

$$
\boldsymbol{\sigma}=J^{-1} \mathbf{F} \cdot \mathbf{S} \cdot \mathbf{F}^{T}
$$

where $\mathbf{F}$ and $J$ are the deformation gradient tensor and its determinant, and $\mathbf{S}$ is the second Piola-Kirchhoff stress tensor. It is obtained from the strain-energy density function $\varphi$ as follows:

$$
\mathbf{S} \equiv \frac{\partial \varphi}{\partial \mathbf{E}}
$$


where $\mathbf{E}$ is the Green-Lagrange strain tensor:

$$
\mathbf{E}=\frac{1}{2}(\mathbf{C}-\mathbf{I})
$$

and $\mathbf{C}$ is the Cauchy-Green deformation tensor:

$$
\mathbf{C} \equiv \mathbf{F}^{T} \cdot \mathbf{F} .
$$

From Eqs. (5) and (6),

$$
\mathbf{S}=2 \frac{\partial \varphi}{\partial \mathbf{C}}
$$

\subsection{Fluid-structure interface}

In an FSI problem, at the fluid-structure interface, we will have the velocity and stress compatibility conditions between the fluid and structure parts. The details on those conditions can be found in Section 5.1 of [19].

\section{ST-VMS and ST-SUPS}

The ST-VMS and ST-SUPS are versions of the Deforming-Spatial-Domain/Stabilized ST (DSD/SST) method [20-22], which was introduced for computation of flows with moving boundaries and interfaces (MBI), including FSI. The ST-SUPS is a new name for the original version of the DSD/SST, with "SUPS" reflecting its stabilization components, the Streamline-Upwind/Petrov-Galerkin (SUPG ) [23] and Pressure-Stabilizing/PetrovGalerkin (PSPG) [20] stabilizations. The STVMS is the VMS version of the DSD/SST. The VMS components of the ST-VMS are from the residual-based VMS (RBVMS) method [2427]. The five stabilization terms of the STVMS include the three that the ST-SUPS has, and therefore the ST-VMS subsumes the STSUPS. In MBI computations the ST-VMS and ST-SUPS function as a moving-mesh methods. Moving the fluid mechanics mesh to follow an interface enables mesh-resolution control near the interface and, consequently, high-resolution boundary-layer representation near fluid-solid interfaces. Because of the higher-order accuracy of the ST framework (see $[6,28]$ ), the ST-SUPS and ST-VMS are desirable also in computations without MBI.

The ST-SUPS and ST-VMS have been applied to many classes of challenging FSI, MBI and fluid mechanics problems (see [29] for a comprehensive summary of the computations prior to July 2018). The classes of problems include spacecraft parachute analysis for the landing-stage parachutes [12, 19, 30-32], cover-separation parachutes [33] and the drogue parachutes [34-36], wind-turbine aerodynamics for horizontal-axis wind-turbine rotors [19, 37-39], full horizontal-axis wind-turbines [4043] and vertical-axis wind-turbines [1, 4, 44], flapping-wing aerodynamics for an actual locust [8, 19, 45, 46], bioinspired MAVs [41, 42, 47, 48] and wing-clapping $[49,50]$, blood flow analysis of cerebral aneurysms [41,51], stent-blocked aneurysms [51-53], aortas [54-58], heart valves $[42,49,56,58-64]$ and coronary arteries in motion [65], spacecraft aerodynamics [33,66], thermofluid analysis of ground vehicles and their tires [60,67], thermo-fluid analysis of disk brakes [7], flow-driven string dynamics in turbomachinery $[3,68,69]$, flow analysis of turbocharger turbines [9-11,70,71], flow around tires with road contact and deformation [60,72-75], fluid films [75,76], ram-air parachutes [77], and compressible-flow spacecraft parachute aerodynamics [78,79].

For more on the ST-VMS and ST-SUPS, see [19]. In the flow analyses presented here, the ST framework provides higher-order accuracy in a general context. The VMS feature of the STVMS addresses the computational challenges associated with the multiscale nature of the unsteady flow. The moving-mesh feature of the ST framework enables high-resolution computation near the rotor surface. The advection equation involved in the residence time computation associated with flow-driven string dynamics in pumps is solved with the ST-SUPG method.

\section{ALE-VMS, RBVMS and ALE-SUPS}

The ALE-VMS $[5,19,80-84]$ is the VMS version of the ALE [85]. It succeeded the STSUPS [20] and ALE-SUPS [86] and preceded 
the ST-VMS. The VMS components are from the RBVMS [24-27]. It is the moving-mesh extension of the RBVMS formulation of incompressible turbulent flows proposed in [26], and as such, it was first presented in [5] in the FSI context. The ALE-SUPS, RBVMS and ALEVMS have also been applied to many classes of challenging FSI, MBI and fluid mechanics problems. The classes of problems include ram-air parachute FSI [86], wind-turbine aerodynamics and FSI [4, 37, 43, 44, 87-93], more specifically, vertical-axis wind turbines $[4,44,94,95]$, floating wind turbines [96], wind turbines in atmospheric boundary layers [4, 44, 93, 97], and fatigue damage in wind-turbine blades [2], patientspecific cardiovascular fluid mechanics and FSI [5, 98-103], biomedical-device FSI [104-109], ship hydrodynamics with free-surface flow and fluid-object interaction [110,111], hydrodynamics and FSI of a hydraulic arresting gear [112, 113], hydrodynamics of tidal-stream turbines with free-surface flow [114], passive-morphing FSI in turbomachinery [115], bioinspired FSI for marine propulsion [116,117], bridge aerodynamics and fluid-object interaction [118-120], and mixed ALE-VMS/Immersogeometric computations [107-109, 121, 122] in the framework of the Fluid-Solid Interface-Tracking/InterfaceCapturing Technique [123]. Recent advances in stabilized and multiscale methods may be found for stratified incompressible flows in [124], for divergence-conforming discretizations of incompressible flows in [125], and for compressible flows with emphasis on gas-turbine modeling in [126].

For more on the ALE-VMS, RBVMS and ALE-SUPS, see [19]. In the flow analyses presented here, the VMS feature of the ALE-VMS addresses the computational challenges associated with the multiscale nature of the unsteady flow. The moving-mesh feature of the ALE framework enables high-resolution computation near the rotor surface.

\section{ALE-SI and ST-SI}

The ALE-SI was introduced in $[16,17]$ to retain the desirable moving-mesh features of the ALEVMS in computations with spinning solid sur- faces, such as a turbine rotor. The mesh covering the spinning surface spins with it, retaining the high-resolution representation of the boundary layers. The method was in the context of incompressible-flow equations. Interface terms added to the ALE-VMS to account for the compatibility conditions for the velocity and stress at the SI accurately connect the two sides of the solution. The ST-SI was introduced in [1], also in the context of incompressible-flow equations, to retain the desirable moving-mesh features of the ST-VMS and ST-SUPS in computations with spinning solid surfaces. The starting point in its development was the ALE-SI. Interface terms similar to those in the ALE-SI are added to the ST-VMS to accurately connect the two sides of the solution. An ST-SI version where the SI is between fluid and solid domains was also presented in [1]. The SI in this case is a "fluid-solid SI" rather than a standard "fluid-fluid ST" and enables weak enforcement of the Dirichlet boundary conditions for the fluid. The ST-SI introduced in [7] for the coupled incompressible-flow and thermal-transport equations retains the high-resolution representation of the thermo-fluid boundary layers near spinning solid surfaces. These ST-SI methods have been applied to aerodynamic analysis of vertical-axis wind turbines $[1,4,44]$, thermo-fluid analysis of disk brakes [7], flow-driven string dynamics in turbomachinery [3,68,69], flow analysis of turbocharger turbines [9-11,70,71], flow around tires with road contact and deformation [60,72-75], fluid films [75,76], aerodynamic analysis of ram-air parachutes [77], and flow analysis of heart valves [56, 58,61-64].

For more on the ST-SI, see $[1,7]$. In the computations here, with the ALE-SI and ST-SI the mesh covering the rotor spins with it and we retain the high-resolution representation of the boundary layers.

\section{Stabilization parameters}

The ST-SUPS, ALE-SUPS, RBVMS, ALE-VMS and ST-VMS all have some embedded stabilization parameters that play a significant role 
(see [19]). These parameters involve a measure of the local length scale (also known as "element length") and other parameters such as the element Reynolds and Courant numbers. There are many ways of defining the stabilization parameters. Some of the newer options for the stabilization parameters used with the SUPS and VMS can be found in $[1,8,39,40,67,74,127-130]$. Some of the earlier stabilization parameters used with the SUPS and VMS were also used in computations with other SUPG-like methods, such as the computations reported in [115, 131-142]. The stabilization-parameter definitions used in the computations reported in this article can be found from the references cited in the sections where those computations are described.

\section{ST-IGA}

The ST-IGA is the integration of the ST framework with isogeometric discretization, motivated by the success of NURBS meshes in spatial discretization $[5,16,98,143]$. It was introduced in [6]. Computations with the ST-VMS and STIGA were first reported in [6] in a $2 \mathrm{D}$ context, with IGA basis functions in space for flow past an airfoil, and in both space and time for the advection equation. Using higher-order basis functions in time enables getting full benefit out of using higher-order basis functions in space (see the stability and accuracy analysis given in [6] for the advection equation).

The ST-IGA with IGA basis functions in time enables, as pointed out and demonstrated in $[6,8,28,45,47]$, a more accurate representation of the motion of the solid surfaces and a mesh motion consistent with that. It also enables more efficient temporal representation of the motion and deformation of the volume meshes, and more efficient remeshing. These motivated the development of the STNMUM $[8,40,45,47]$. The STNMUM has a wide scope that includes spinning solid surfaces. With the spinning motion represented by quadratic NURBS in time, and with sufficient number of temporal patches for a full rotation, the circular paths are represented exactly. A "secondary mapping" $[6,8,19,28]$ enables also specifying a constant angular velocity for invariant speeds along the circular paths.
The ST framework and NURBS in time also enable, with the "ST-C" method, extracting a continuous representation from the computed data and, in large-scale computations, efficient data compression $[3,7,60,67-69,144]$. The STNMUM and the ST-IGA with IGA basis functions in time have been used in many $3 \mathrm{D}$ computations. The classes of problems solved are flapping-wing aerodynamics for an actual locust $[8,19,45,46]$, bioinspired MAVs $[41,42,47,48]$ and wing-clapping $[49,50]$, separation aerodynamics of spacecraft [33], aerodynamics of horizontalaxis [40-43] and vertical-axis [1, 4, 44] windturbines, thermo-fluid analysis of ground vehicles and their tires [60,67], thermo-fluid analysis of disk brakes [7], flow-driven string dynamics in turbomachinery $[3,68,69]$, flow analysis of turbocharger turbines [9-11,70,71], and flow analysis of coronary arteries in motion [65].

The ST-IGA with IGA basis functions in space enables more accurate representation of the geometry and increased accuracy in the flow solution. It accomplishes that with fewer control points, and consequently with larger effective element sizes. That in turn enables using larger time-step sizes while keeping the Courant number at a desirable level for good accuracy. It has been used in ST computational flow analysis of turbocharger turbines [9-11,70,71], flow-driven string dynamics in turbomachinery [3,69], ramair parachutes [77], spacecraft parachutes [79], aortas [56-58], heart valves [56, 58,61-64], coronary arteries in motion [65], tires with road contact and deformation [73-75], and fluid films $[75,76]$. Using IGA basis functions in space is now a key part of some of the newest arterial zero-stress-state (ZSS) estimation methods [58,145-150] and related shell analysis [151].

For more on the ST-IGA, see $[9,19,45,77]$. In the computational flow analyses presented here, the ST-IGA enables more accurate representation of the turbine and turbomachinery geometries, increased accuracy in the flow solution, and using larger time-step sizes. Integration of the ST-SI with the ST-IGA enables a more accurate representation of the rotor motion and a mesh motion consistent with that, and we will describe the ST-SI-IGA in Section 8. 


\section{ST-SI-IGA}

The ST-SI-IGA is the integration of the STSI and ST-IGA. The turbocharger turbine flow [9-11, 70, 71] and flow-driven string dynamics in turbomachinery $[3,69]$ were computed with the ST-SI-IGA. The IGA basis functions were used in the spatial discretization of the fluid mechanics equations and also in the temporal representation of the rotor and spinning-mesh motion. That enabled accurate representation of the turbine geometry and rotor motion and increased accuracy in the flow solution. The IGA basis functions were used also in the spatial discretization of the string structural dynamics equations. That enabled increased accuracy in the structural dynamics solution, as well as smoothness in the string shape and fluid dynamics forces computed on the string.

The ram-air parachute analysis [77] and spacecraft parachute compressible-flow analysis [79] were conducted with the ST-SI-IGA, based on the ST-SI version that weakly enforces the Dirichlet conditions and the ST-SI version that accounts for the porosity of a thin structure. The ST-IGA with IGA basis functions in space enabled, with relatively few number of unknowns, accurate representation of the parafoil and parachute geometries and increased accuracy in the flow solution. The volume mesh needed to be generated both inside and outside the parafoil. Mesh generation inside was challenging near the trailing edge because of the narrowing space. The spacecraft parachute has a very complex geometry, including gores and gaps. Using IGA basis functions addressed those challenges and still kept the element density near the trailing edge of the parafoil and around the spacecraft parachute at a reasonable level.

In the heart valve analysis [56,58,61-64], the ST-SI-IGA, beyond enabling a more accurate representation of the geometry and increased accuracy in the flow solution, kept the element density in the narrow spaces near the leaflet contact areas at a reasonable level.

In computational analysis of flow around tires with road contact and deformation [73-75], the ST-SI-IGA enables a more accurate representation of the geometry and motion of the tire sur- faces, a mesh motion consistent with that, and increased accuracy in the flow solution. It also keeps the element density in the tire grooves and in the narrow spaces near the contact areas at a reasonable level. In addition, we benefit from the mesh generation flexibility provided by using SIs. In computational analysis of fluid films [75, 76], the ST-SI-IGA enabled solution with a computational cost comparable to that of the Reynolds-equation model for the comparable solution quality [76]. With that, narrow gaps associated with the road roughness [75] can be accounted for in the flow analysis around tires.

An SI provides mesh generation flexibility in a general context by accurately connecting the two sides of the solution computed over nonmatching meshes. This type of mesh generation flexibility is especially valuable in complex-geometry flow computations with isogeometric discretization, removing the matching requirement between the NURBS patches without loss of accuracy. This feature was used in the flow analysis of heart valves [56,58,61-64], turbocharger turbines [9-11, 70,71], and spacecraft parachute compressible-flow analysis [79].

For more on the ST-SI-IGA, see [77]. In the computations presented here, the ST-SI-IGA is used for the reasons given and as described in the first paragraph of this section.

\section{General-purpose NURBS mesh generation method}

While the IGA provides superior accuracy and high-fidelity solutions, to make its use even more practical in computational flow analysis with complex geometries, NURBS volume mesh generation needs to be easier and more automated. The general-purpose NURBS mesh generation method introduced in [10] serves that purpose. The method is based on multi-block-structured mesh generation with established techniques, projection of that mesh to a NURBS mesh made of patches that correspond to the blocks, and recovery of the original model surfaces. The recovery of the original surfaces is to the extent 
they are suitable for accurate and robust computations. The method targets retaining the refinement distribution and element quality of the multi-block-structured mesh that we start with. Because good techniques and software for generating multi-block-structured meshes are easy to find, the method makes general-purpose NURBS mesh generation relatively easy.

Mesh-quality performance studies for $2 \mathrm{D}$ and $3 \mathrm{D}$ meshes, including those for complex models, were presented in [11]. A test computation for a turbocharger turbine and exhaust manifold was also presented in [11], with a more detailed computation in [70]. The mesh generation method was used also in the pump-flow analysis part of the flow-driven string dynamics presented in [3] and in the aorta flow analysis presented in $[56,57]$. The performance studies, test computations and actual computations demonstrated that the general-purpose NURBS mesh generation method makes the IGA use in fluid mechanics computations even more practical.

For more on the general-purpose NURBS mesh generation method, see $[10,11]$. In the computations presented here, the method is used for the VAWT and for the pump-flow part of the flow-driven string dynamics.

\section{Other computational methods}

\subsection{String dynamics}

The string in the flow-driven string dynamics is modeled with bending-stabilized cable elements [152], using the IGA with cubic NURBS basis functions. This gives us a higher-order method, and smoothness in the structure shape. It also gives us smoothness in the fluid forces acting on the string. Because a string is a very thin structure, its influence on the flow will be very small. In the one-way-dependence model, we compute the influence of the flow on the string dynamics, while avoiding the formidable task of computing the influence of the string on the flow. The fluid mechanics forces acting on the string are calculated with the method described in [12] for computing the aerodynamic forces acting on the sus- pension lines of spacecraft parachutes. Contact between the string and solid surfaces is handled with the Surface-Edge-Node Contact Tracking (SENCT-FC) method [153], which is a later version of the SENCT introduced in [22].

\subsection{Particle residence time}

In flow-driven string dynamics in pumps, the residence time computations help us to have a simplified but quick understanding of the string behavior. The computation is based on solving a time-dependent advection equation with a unit source term. For more on the computation method, see [3].

\subsection{Rotation representation with constant angular velocity}

We use quadratic NURBS functions, as described in [8], to represent a circular-arc trajectory. The secondary mapping concept, introduced in [6], enables us specify a constant velocity along that trajectory. For more on this method, see $[6,8]$.

\section{ST computation: flow-driven string dynamics in a pump}

This section is from [3].

\subsection{Flow analysis of the pump}

We use a vortex pump with 6 blades, including two higher-height blades. The rotor diameter is roughly $150 \mathrm{~mm}$. We are unable to provide more details due to the industrial-partner restrictions. The quadratic NURBS mesh used in the computation is shown in Figure 1. The number of control points and elements are 838,222 and 544,466. The pump is used for water, the density is $998.2 \mathrm{~kg} / \mathrm{m}^{3}$, and the kinematic viscosity $8.7 \times 10^{-7} \mathrm{~m}^{2} / \mathrm{s}$. The rotation speed is 
2,544 rpm. The boundary conditions are shown in Figure 2.
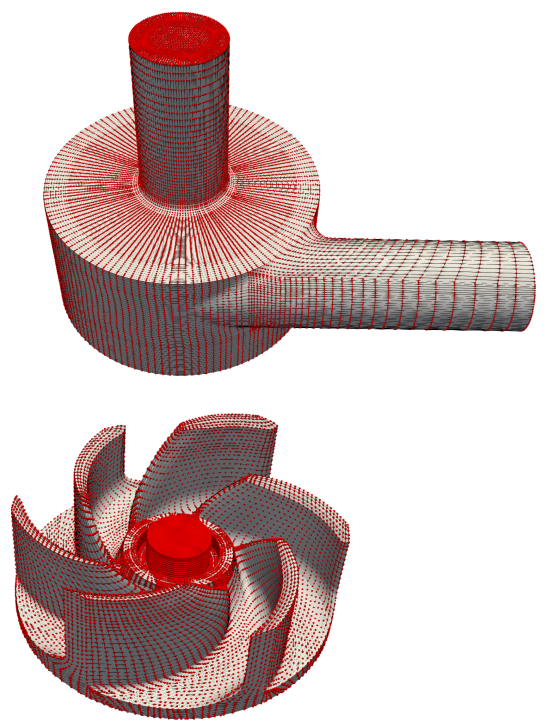

Fig. 1: Control mesh. Red circles represent the control points.

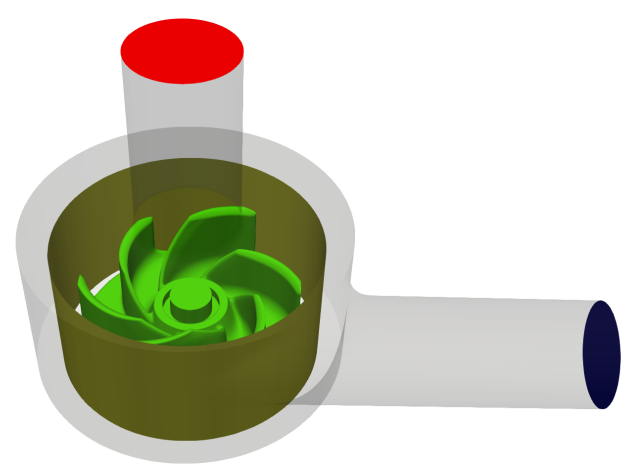

Fig. 2: Boundary conditions. Flow velocity at the inlet (red), zero-stress at the outlet (blue), and noslip on the wall and rotor (green). The circular interface (yellow) is the SI.

At the inlet, $Q=5.46 \times 10^{-3} \mathrm{~m}^{3} / \mathrm{s}$. The timestep size is $9.8 \times 10^{-5} \mathrm{~s}$. The number of nonlinear iterations per time step is 3 , and the number of GMRES iterations per nonlinear iteration is 100. Stabilization parameters of the ST-VMS are those given by Eqs. (2.4)-(2.6), (2.8) and (2.10) in [1].
Figure 3 shows the second invariant of the velocity gradient tensor. The turbulent nature of the flow is well represented. The solution is compared to the experimental data from Professor Kazuyoshi Miyagawa's group (Waseda University). The conditions here are close to those corresponding to the best-efficiency operating point, and the relative error in the efficiency compared to the experimental data is less than $1.5 \%$. The computed flow field from rotations 17 through 21 is stored with the ST-C [144] as the data compression method and is used repeatedly in the string dynamics and residence time computations.

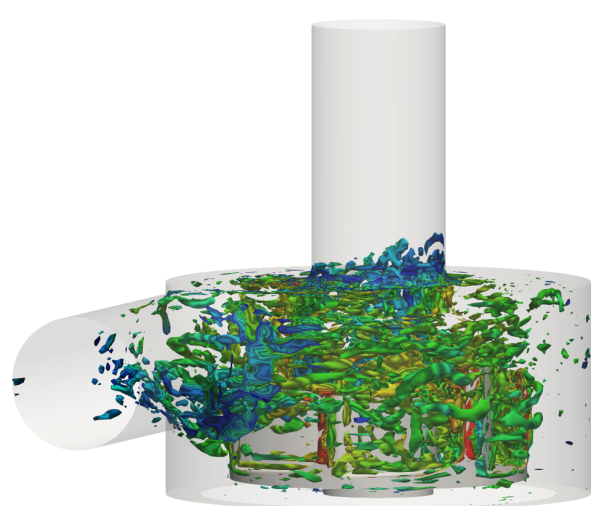

$t=0.094 \mathrm{~s}$

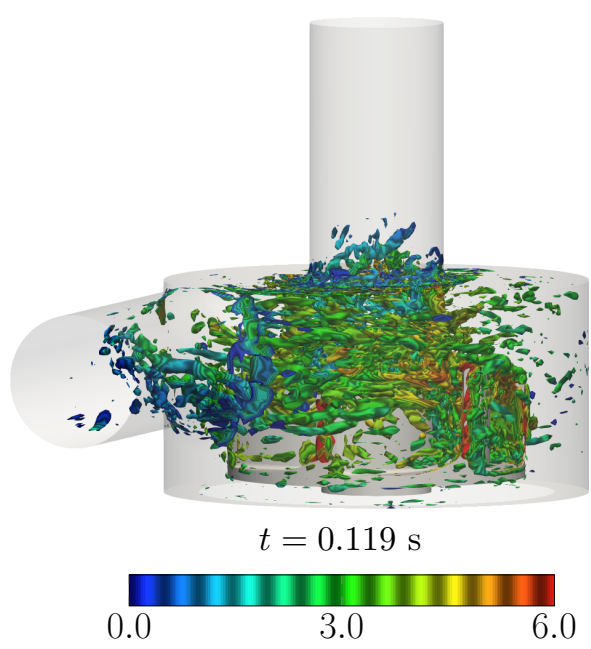

Fig. 3: Isosurfaces of the second invariant value of velocity gradient tensor, colored by the velocity magnitude $(\mathrm{m} / \mathrm{s})$. 


\subsection{String dynamics in the pump}

The string has $1.5 \mathrm{~mm}$ diameter and circularshape cross-section. We compute with three different string lengths, 10, 50 and $70 \mathrm{~mm}$. The Young's modulus and density are 5.0 MPa and $960 \mathrm{~kg} / \mathrm{m}^{3}$. We use a cubic NURBS mesh, with 19 control points and 16 elements. There are 17 different initial positions, shown in Figure 4. The initial string velocity is $2.0 \mathrm{~m} / \mathrm{s}$, in the

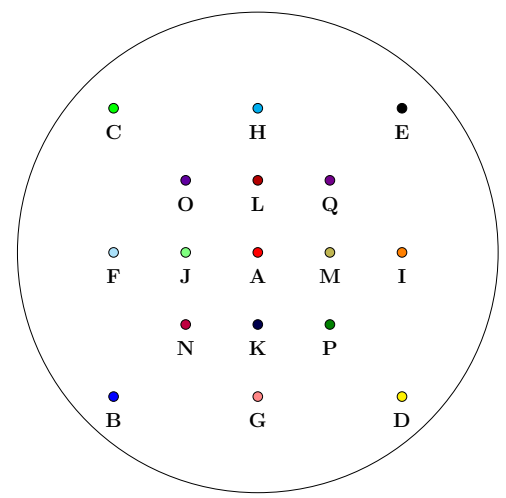

Fig. 4: The initial positions of the strings at the inlet plane.

flow direction. The time-step size is $9.8 \times 10^{-4} \mathrm{~s}$, which is 10 times smaller than the time-step size used in the flow computation. The number of nonlinear iterations per time step is 3 , with full GMRES (i.e. until no more Krylov vectors can be found).

Figures $5-7$ show, for the three different string lengths, the string with the initial position at $\mathrm{A}$ (see Figure 4). In all three cases the string first hits the top of the blade, and then moves to the edge of the pump casing.

\subsection{Residence time for the pump}

The computation is carried out with a time-step size of $4.9 \times 10^{-4} \mathrm{~s}$, which is 5 times larger than the time-step size used in the flow computation. The number of nonlinear iterations per time step is 2, and the number of GMRES iterations per nonlinear iteration is 30 .
The flow-rate-averaged residence time over the outlet is shown in Figure 8. After $1.2 \mathrm{~s}$ it reaches the maximum value. Figure 9 shows the spatial distribution of the residence time at the end of the computation. The residence time under the rotor is much higher than the residence time at the outlet, which is around $0.4 \mathrm{~s}$. This means that this region is not connected to the main flow.

\subsection{Discussion}

We discuss the relationship between the string dynamics and the residence time. Figure 10 show, for the string with length $70 \mathrm{~mm}$, the time histories of the string centroid positions in radius and height. We see some strings moving in circles along the bottom edges of the casing. These strings tend to stay there and cannot rise up. Therefore they stay in the pump forever. This can be correlated with the high residence time at the bottom of the pump (Figure 9).

\section{ST computation: aerodynamics of a VAWT}

We present our preliminary test computations with $2 \mathrm{D}$ model of the aerodynamics of a VAWT. The wind turbine has four support columns at the periphery. Figure 11 shows the wind turbine. The design is modeled after the wind turbine in [154]. The rotor diameter is $16 \mathrm{~m}$, and the machine height is $45 \mathrm{~m}$. The three blades are based on the NACA0015 airfoil, and the cord length and the blade height are $1.5 \mathrm{~m}$ and $18 \mathrm{~m}$, respectively. There are two connecting rods from the hub to each blade, and the blades are supported without any tilt with respect to the tangent of the rotation path. The four support columns are cylindrical with circular cross-section, and they provide enough strength to support the rotor, which is estimated to weigh $3 \mathrm{t}$.

We carry out the computations at a constant free-stream velocity $U_{\infty}$ and with prescribed rotor motion at constant angular velocity. The rotation is clockwise viewed from the top. The air 




$$
t=0 \mathrm{~s}
$$



$$
t=0.060 \mathrm{~s}
$$



$$
t=0.131 \mathrm{~s}
$$

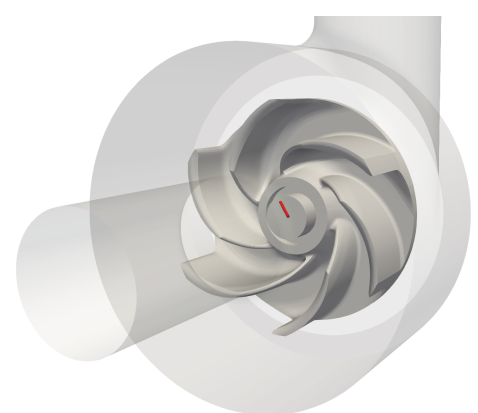

$$
t=0.039 \mathrm{~s}
$$
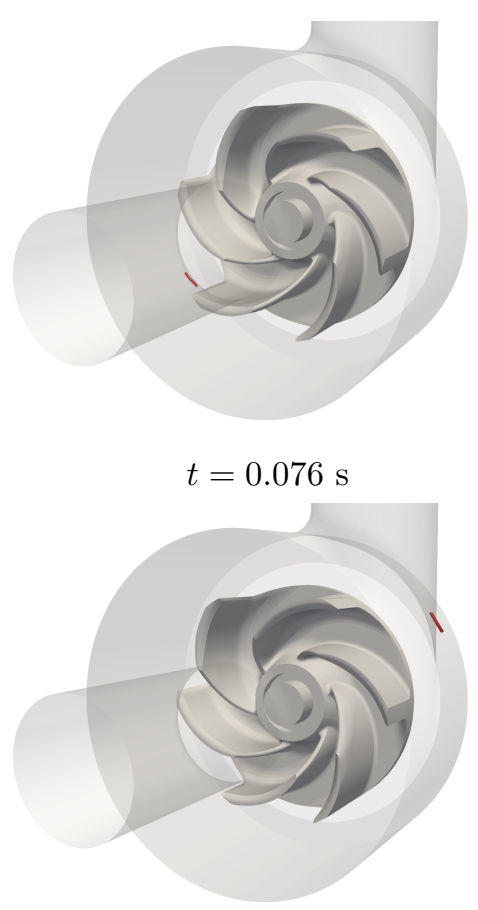

$t=0.187 \mathrm{~s}$

Fig. 5: String with length $10 \mathrm{~mm}$ and initial position at A. 




$t=0 \mathrm{~s}$

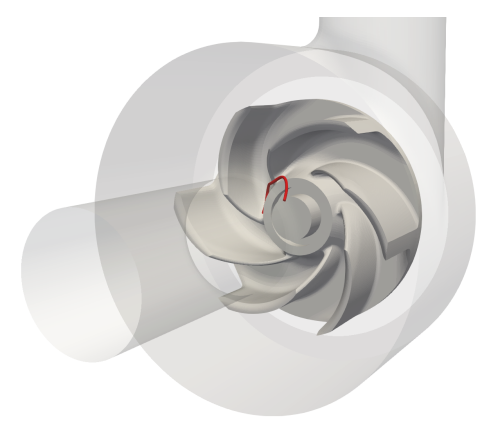

$t=0.060 \mathrm{~s}$

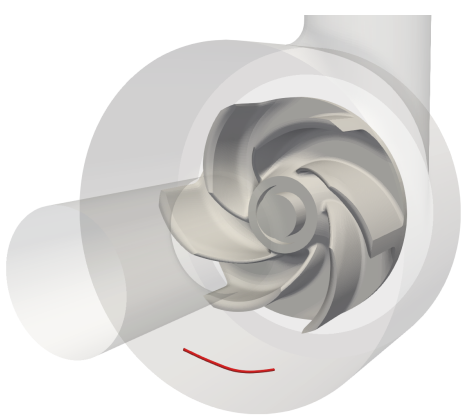

$$
t=0.131 \mathrm{~s}
$$

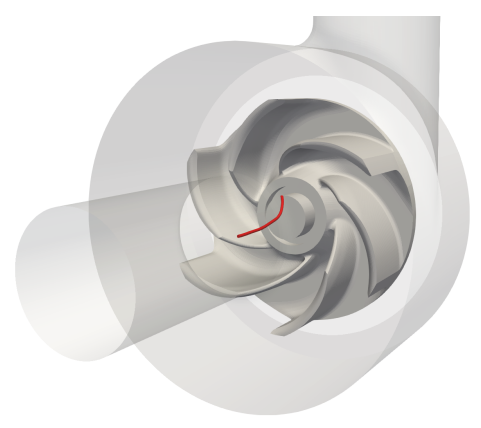

$t=0.039 \mathrm{~s}$

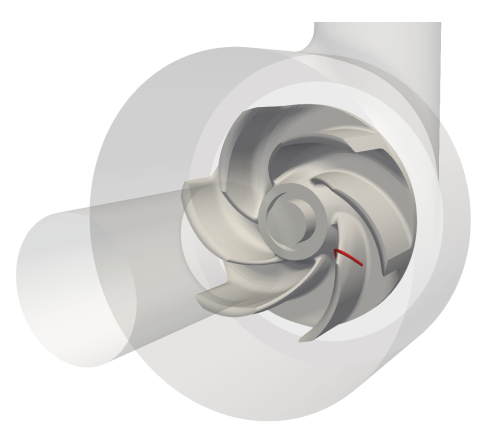

$t=0.076 \mathrm{~s}$

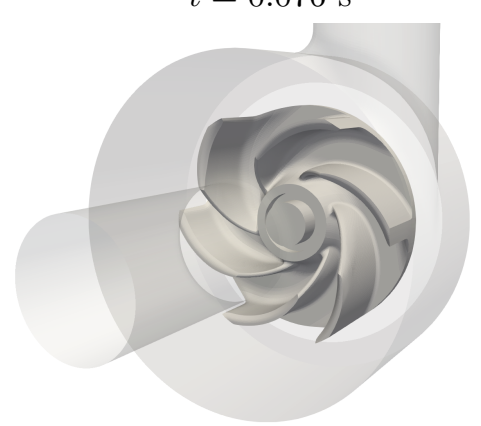

$t=0.187 \mathrm{~s}$

Fig. 6: String with length $50 \mathrm{~mm}$ and initial position at A. We note that the string leaves the casing before the 6 th picture. 


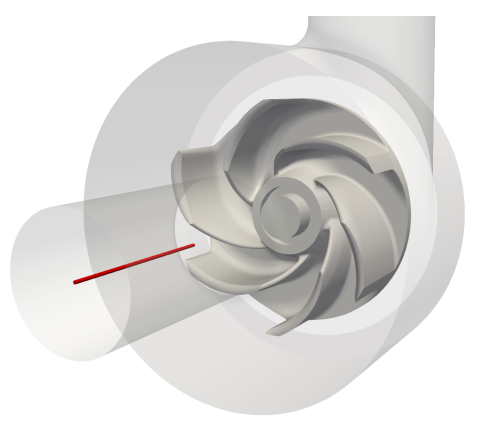

$t=0 \mathrm{~s}$

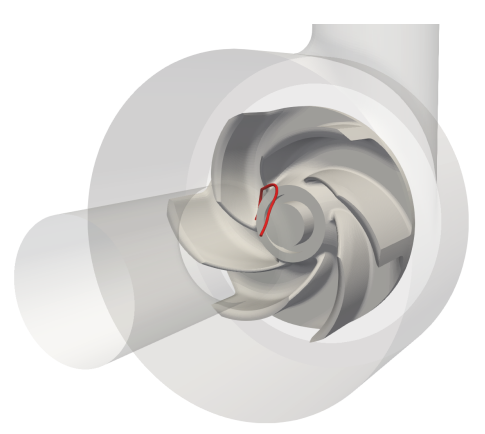

$$
t=0.060 \mathrm{~s}
$$

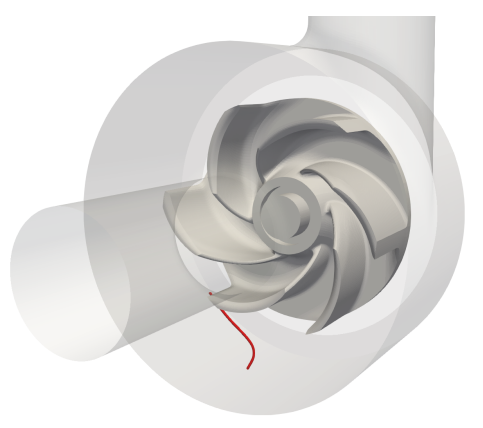

$t=0.131 \mathrm{~s}$

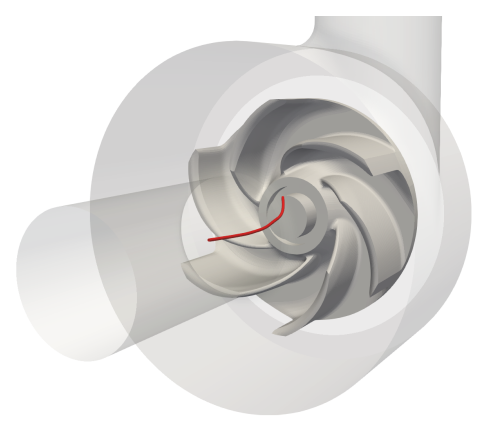

$t=0.039 \mathrm{~s}$



$t=0.076 \mathrm{~s}$

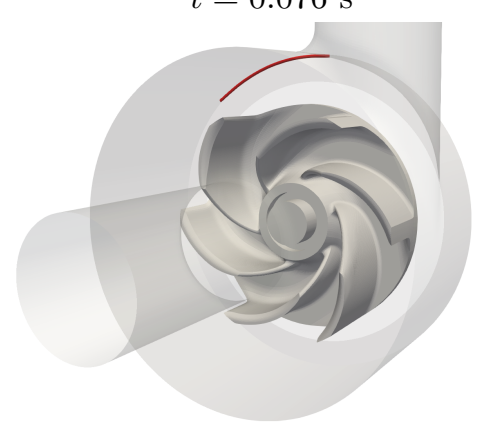

$t=0.187 \mathrm{~s}$

Fig. 7: String with length $70 \mathrm{~mm}$ and initial position at A. 


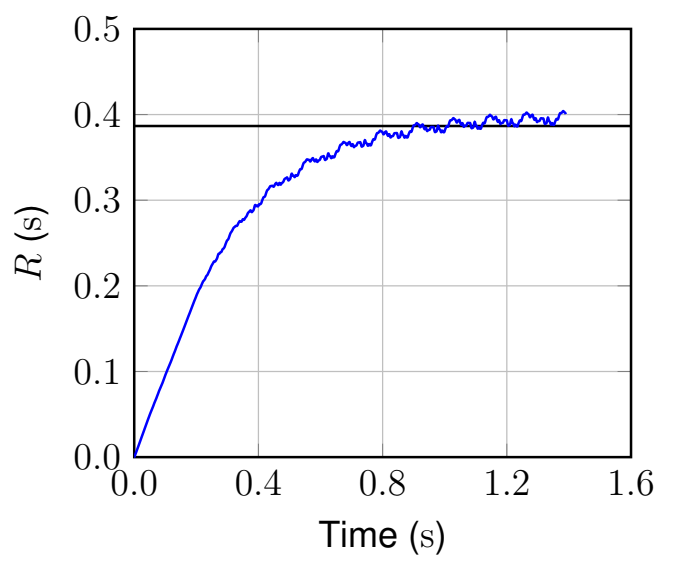

_ Theoretical value _ Computed value

Fig. 8: Flow-rate-averaged residence time over the outlet. Computed value $\left(\bar{R}_{\text {out }}\right)$ and theoretical value $(V / Q)$.

density and kinematic viscosity are $1.205 \mathrm{~kg} / \mathrm{m}^{3}$ and $1.511 \times 10^{-5} \mathrm{~m}^{2} / \mathrm{s}$. We define the blade orientation as represented by the angle $\phi$ seen in Figure 12.

With that orientation, the flow speed seen by a blade can be calculated as

$$
V=U_{\infty} \sqrt{1-2 \lambda \sin \phi+\lambda^{2}},
$$

where $\lambda$ is the tip-speed ratio (TSR). The symbol $T$ will denote the rotation cycle.

The computational-domain size is 62.5 times the rotor diameter in the wind direction, with a distance of 18.75 times the rotor diameter between the upstream boundary and the center of the rotor. In the cross-wind direction, the domain size is 37.5 times the rotor diameter. The mesh position is represented by quadratic NURBS in time. There are three patches that are $120^{\circ}$ each, and the secondary mapping introduced in [8] is used to achieve the constant angular velocity. The free-stream velocity is $12.56 \mathrm{~m} / \mathrm{s}$.

We compute with TSR $=4$. The model geometry and the SI are shown in Figure 13. The boundary conditions are $U_{\infty}$ at the inflow, zero stress at the outflow, slip at the lateral boundaries, and no-slip on the rotor and support column surfaces. The prescribed velocity is evaluated at the integration points, with the values

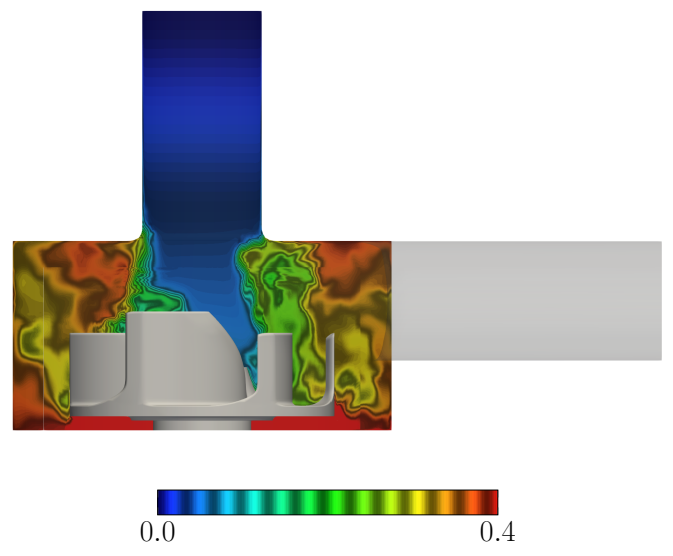

Fig. 9: Residence time (s) on a cut plane at $t=1.297 \mathrm{~s}$, end of the computation.

extracted from the NURBS representation of the rotor surface velocity.

We use two different meshes. We start with Mesh 1, and obtain the other mesh by knot insertion. We halve the knot spacing to get Mesh 2. Figure 14 shows Mesh 1. The number of control points and elements are shown in Table 1 . We

Tab. 1: 2D VAWT. Number of control points $(n c)$ and elements $(n e)$.

\begin{tabular}{l|r|r}
\hline Mesh & $n c$ & $n e$ \\
\hline \hline Mesh 1 & 7,510 & 5,756 \\
\hline Mesh 2 & 26,432 & 23,024 \\
\hline
\end{tabular}

compute with two different time-step sizes. The two time-step sizes selected translate to $\Delta \phi=2^{\circ}$ and $\Delta \phi=1^{\circ}$ per time step. The number of nonlinear iterations per time step is 5 , and the number of GMRES iterations per nonlinear iteration is 300 . The first three nonlinear iterations are based on the ST-SUPS, and the last two the ST-VMS. The stabilization parameters are those given by Eqs. (4)-(8), and (10) in [70]. In the ST-SI, we set $C=2$.

Figures 15 and 16 show, for Mesh 1 with $\Delta \phi=1^{\circ}$ and Mesh 2 with $\Delta \phi=2^{\circ}$, the velocity magnitude in the wake of the support columns located at $\phi=180^{\circ}$ and $\phi=90^{\circ}$. Overall, the wakes are captured better with smaller Courant numbers. 

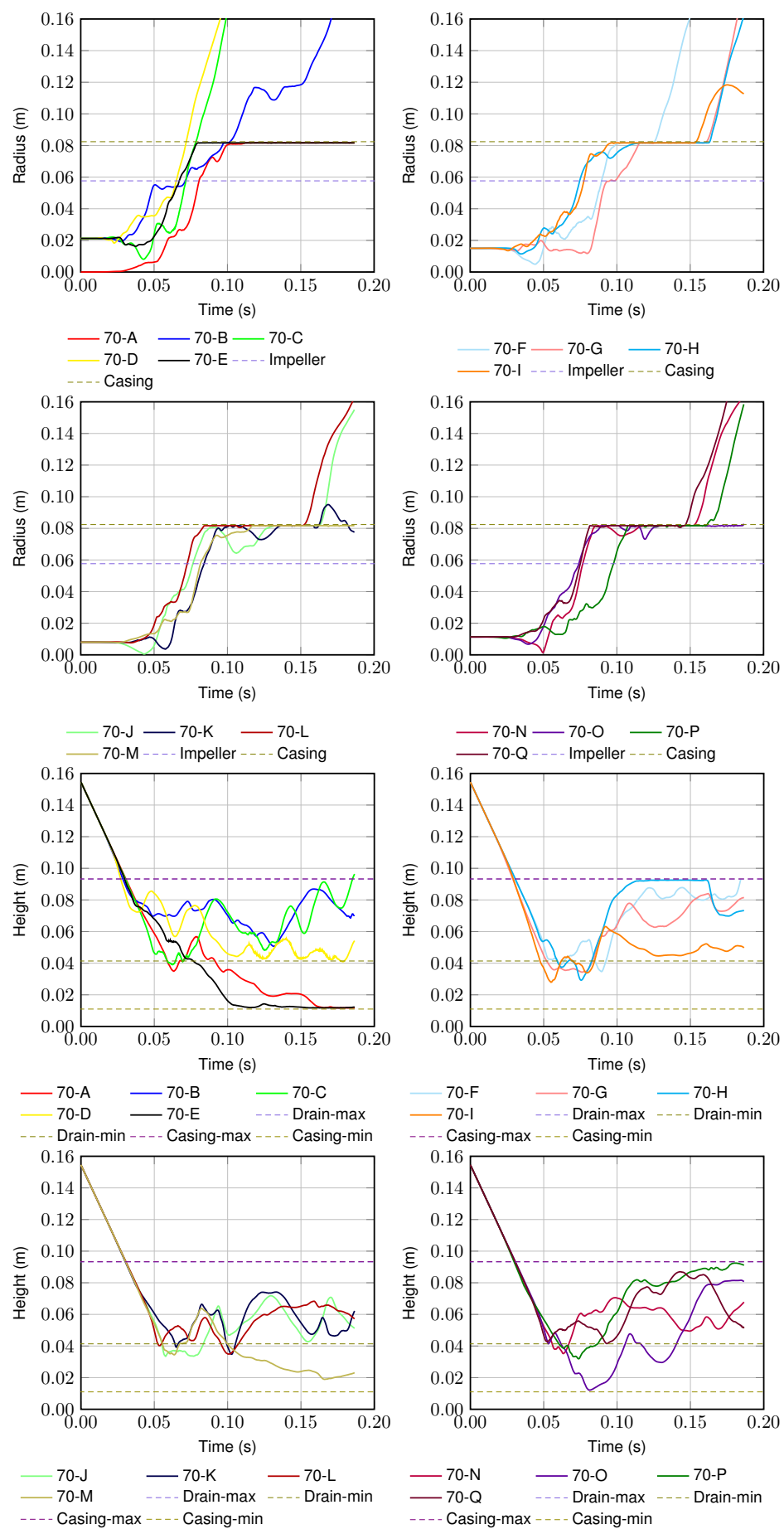

Fig. 10: String with length $70 \mathrm{~mm}$. Time histories of the string centroid positions in radius and height. 


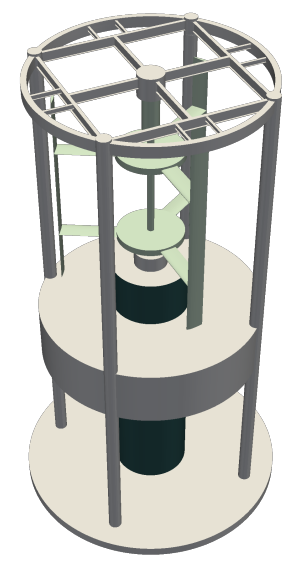

Fig. 11: A VAWT.

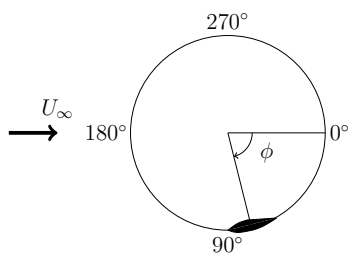

Fig. 12: Blade orientation as represented by the angle

$\phi$.

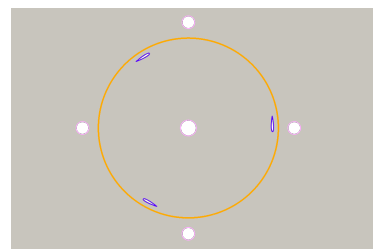

Fig. 13: 2D VAWT. Model geometry and SI.
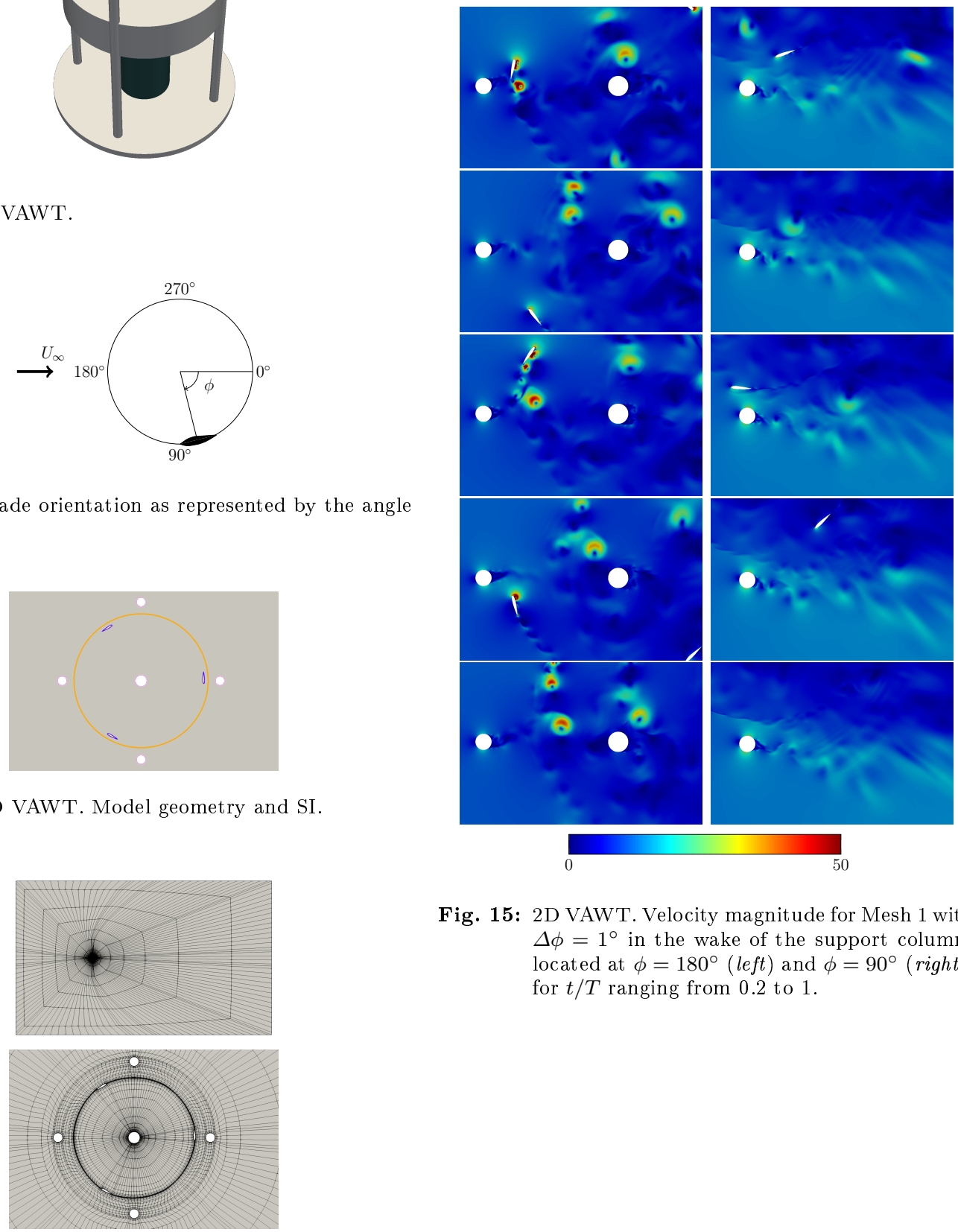

Fig. 15: 2D VAWT. Velocity magnitude for Mesh 1 with $\Delta \phi=1^{\circ}$ in the wake of the support columns located at $\phi=180^{\circ}$ (left) and $\phi=90^{\circ}$ (right), for $t / T$ ranging from 0.2 to 1 .

Fig. 14: 2D VAWT. Mesh 1 (control mesh). 


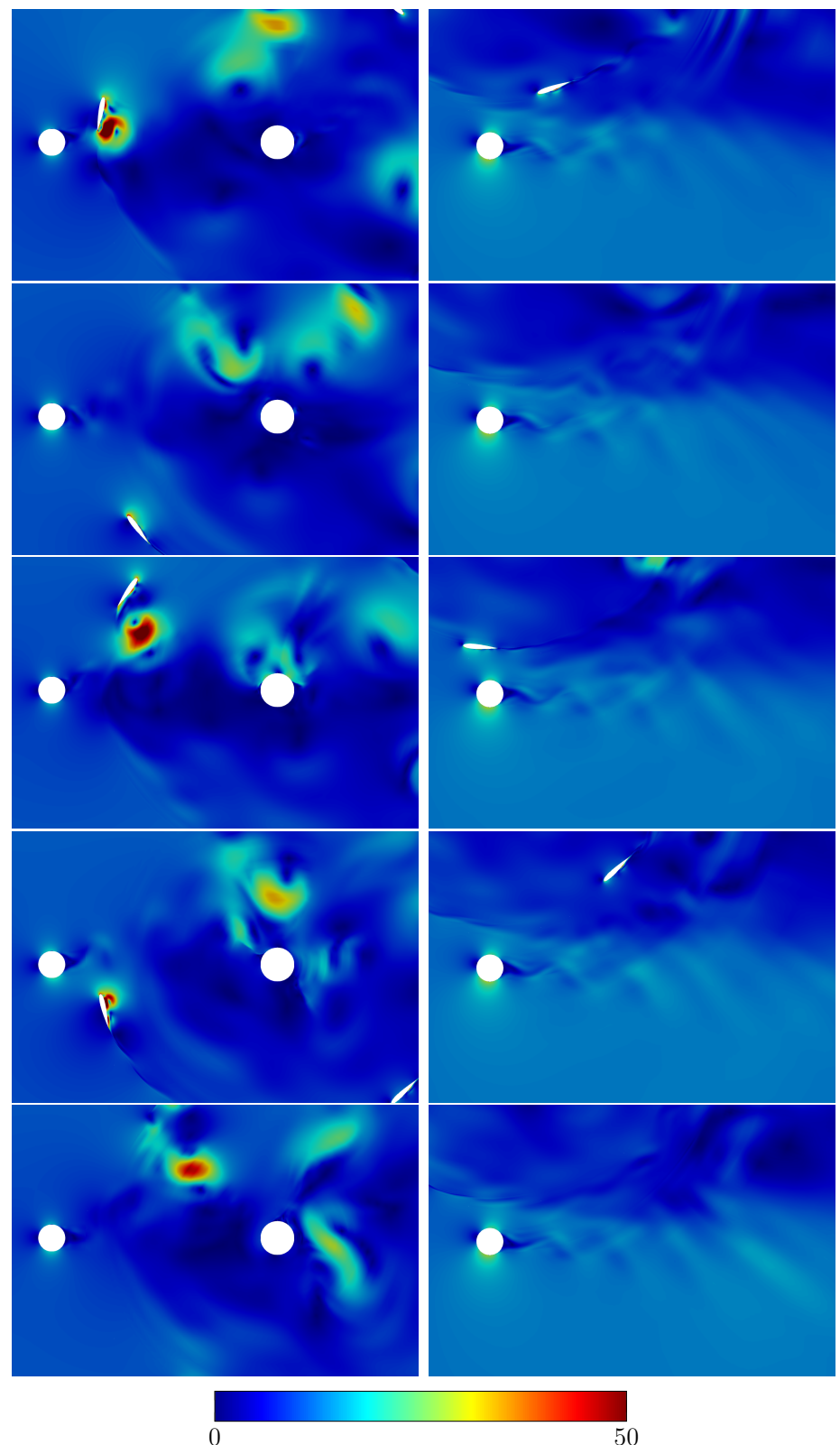

Fig. 16: 2D VAWT. 2D VAWT. Velocity magnitude for Mesh 2 with $\Delta \phi=2^{\circ}$ in the wake of the support columns located at $\phi=180^{\circ}$ (left) and $\phi=90^{\circ}$ (right), for $t / T$ ranging from 0.2 to 1 . 


\section{ALE computation: HAWT FSI with rotor-tower coupling}

Dynamic coupling of a spinning rotor with flexible blades to a deformable tower presents a challenge for standalone structural, as well as coupled FSI simulations. In this section we address this challenge by using a penalty-based approach that allows load transfer between the spinning rotor and tower (see Figure 17). This approach presents an alternative technique to that proposed in [92], and naturally accommodates coupling of distinct structural models (e.g., shells and solids) and discretizations (e.g., finite elements and IGA).

\subsection{Formulation of the rotor-tower penalty coupling}

In a wind turbine, the rotor hub is connected to the nacelle by the main shaft that transfers the rotational motion of the rotor hub to the gearbox. Since we do not wish to model the drivetrain operation directly, a simplified rotortower coupling strategy is required. We develop such a strategy by exploiting a penalty-based technique. For this, we first define the regions on both the rotor and nacelle surfaces that interact with one other, and denote them by $\Gamma_{1}$ (rotor side) and $\Gamma_{2}$ (nacelle side). These regions, which are assumed to have a circular shape, are highlighted using distinct colors in Figure 18. We then design the penalty operator, which precludes all relative motion between $\Gamma_{1}$ and $\Gamma_{2}$ except for relative rotation about the rotor axis. This is achieved, conceptually, by using an overconstrained truss-like system to link the two interaction surfaces. More specifically, the change of distance between a point on one surface and every point on the opposing surface, as shown in Figure 18 (a), is penalized. Figure 18 (b) illustrates all penalized distances between the two surfaces. If the set of current distances (see Figure 18 (c)) is not the same as the set of reference distances (see Figure $18(\mathrm{~b})$ ), the penalty term will produce



Fig. 17: Illustration of rotor and tower structural domains $\Omega_{R}$ and $\Omega_{T}$ and combined deformation accounting for the interaction of $\Omega_{R}$ and $\Omega_{T}$.

forces to keep the current distances the same as the reference distances. The remaining challenge is to remove the forces associated with the relative spinning motion. For this, the distances in the reference configuration are computed from the rotated configuration of the rotor. The latter requires calculation of the total rotation angle $\theta$ (see Figure 18 (d)).

With these considerations, the potential form of the penalty term becomes

$$
\begin{aligned}
\Pi_{p} \equiv & \frac{\beta}{2} \int_{\Gamma_{1}} \int_{\Gamma_{2}} \\
& \left(\left\|\mathbf{x}_{1}-\mathbf{x}_{2}\right\|-\left\|\mathbf{X}_{1}^{r}-\mathbf{X}_{2}^{r}\right\|\right)^{2} \mathrm{~d} \Gamma_{2} \mathrm{~d} \Gamma_{1},
\end{aligned}
$$

where $\beta$ is the penalty constant, $\mathbf{x}_{1}$ and $\mathbf{x}_{2}$ are the current positions of the two interaction surfaces, and $\mathbf{X}_{1}^{r}$ and $\mathbf{X}_{2}^{r}$ are the reference positions of the two interaction surfaces after taking their relative rotation into account. To arrive at the contribution of the penalty term to the weak form of the structural mechanics problem, we take a variation of $\Pi_{p}$ with respect to $\mathbf{x}_{1}$ and $\mathbf{x}_{2}$ to obtain

$$
\begin{aligned}
\delta \Pi_{p}= & \frac{\partial \Pi_{p}}{\partial \mathbf{x}_{1}} \cdot \delta \mathbf{x}_{1}+\frac{\partial \Pi_{p}}{\partial \mathbf{x}_{2}} \cdot \delta \mathbf{x}_{2} \\
=\beta & \int_{\Gamma_{1}} \int_{\Gamma_{2}}\left(\delta \mathbf{x}_{1}-\delta \mathbf{x}_{2}\right) \\
& \left(\left\|\mathbf{x}_{1}-\mathbf{x}_{2}\right\|-\left\|\mathbf{X}_{1}^{r}-\mathbf{X}_{2}^{r}\right\|\right) \\
& \cdot \frac{\mathbf{x}_{1}-\mathbf{x}_{2}}{\left\|\mathbf{x}_{1}-\mathbf{x}_{2}\right\|} \mathrm{d} \Gamma_{2} \mathrm{~d} \Gamma_{1} .
\end{aligned}
$$




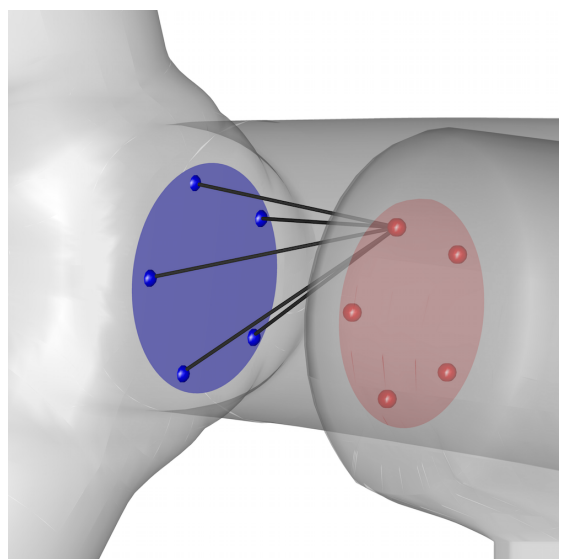

(a) A set of distances between a point on a surface and points on another surface.

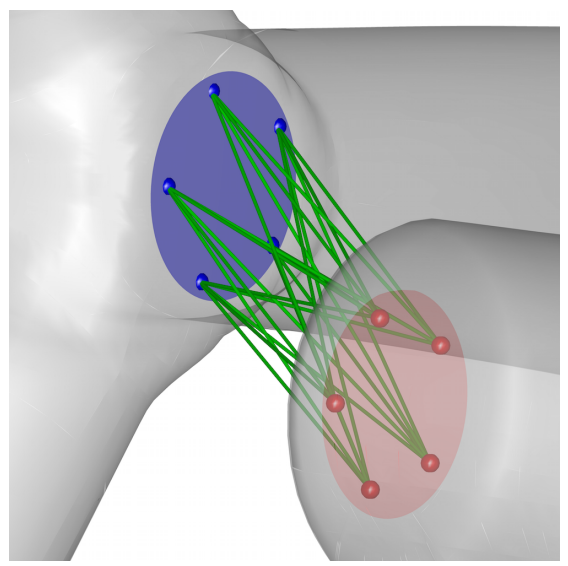

(c) A set of distances in the current configuration.

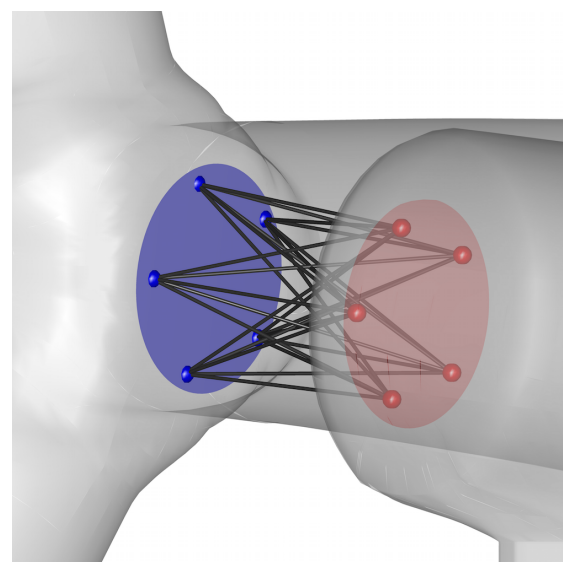

(b) A set of distances in the reference configuration.

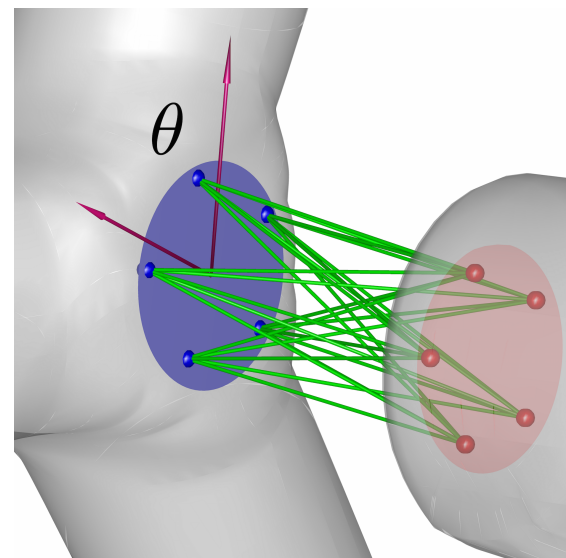

(d) Total rotation angle.

Fig. 18: Key concepts of the penalty-based methodology for rotor-tower coupling.

In the discrete setting, the above integrals are approximated using numerical quadrature. Because only quadrature-point locations and weights are needed to formulate the method, it is well suited for coupling of distinct models and discretizations for the different structural components, which we do in this work.

\subsection{Rotor and tower models and meshes}

A 3D model of the Hexcrete tower is constructed parametrically using the computer-aided design (CAD) software Rhinoceros 3D and the
Grasshopper algorithmic modeling plugin for Rhincoeros (see [155] for details of the parametric modeling methodology). The profile of the tower is hexagonal with smaller hexagonal columns at each corner (see Figure 19).

The tower is comprised of two prismatic sections, located at the top and bottom of the structure, and two intermediate sections with unique rates of taper (see Figure 19). The cylindrical nacelle is also modeled as part of the tower and approximated considered as a solid block. The tower is discretized using 295,332 linear tetrahedral elements. The columns have a Young's modulus $51.36 \mathrm{GPa}$, whereas the panels have a Young's modulus 47.23 GPa. The density and 


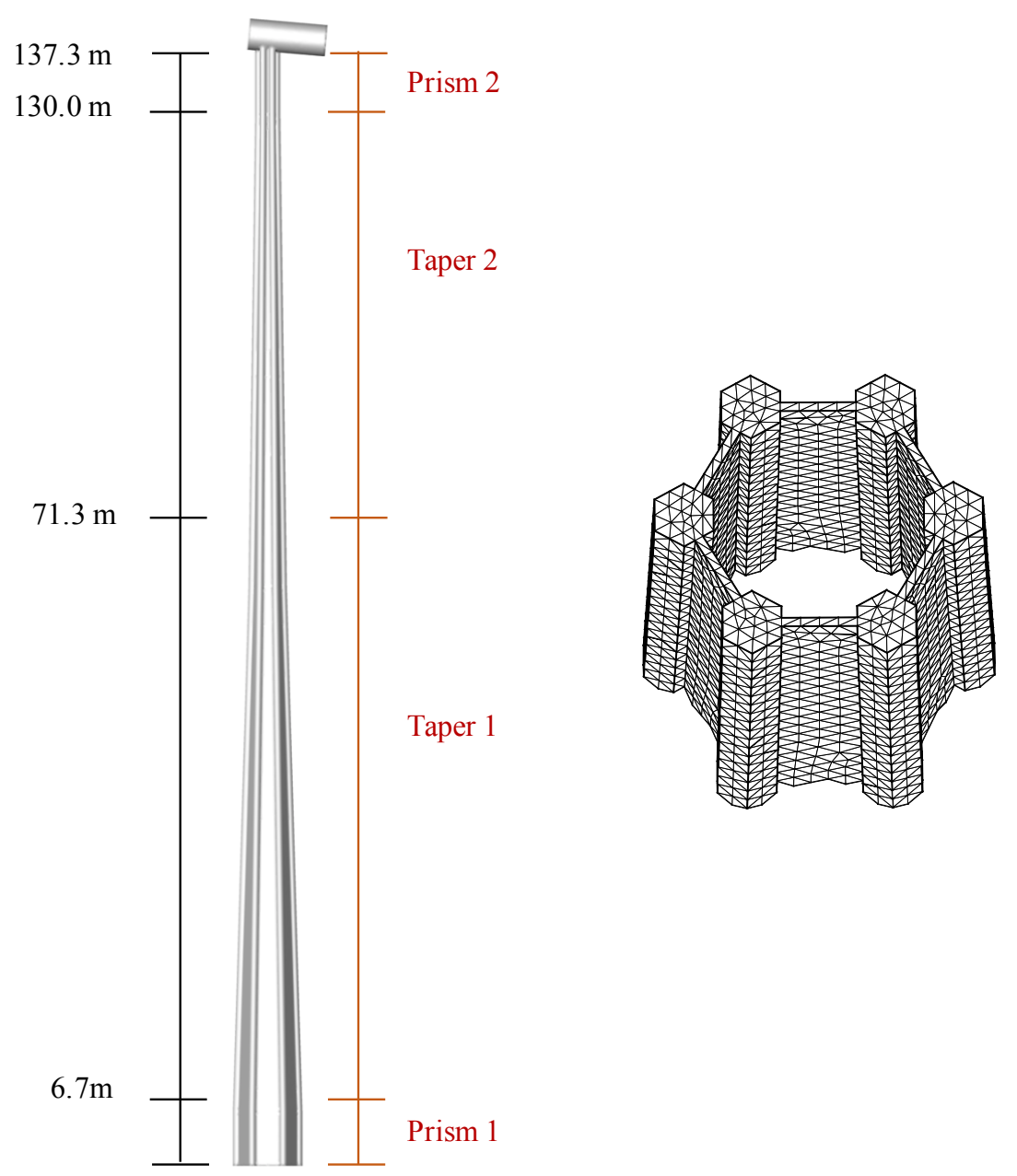

Fig. 19: CAD model of the Hexcrete tower (left) and a section of the tower solid mesh (right).

Poisson's ratio of both are assumed to be 2,392 $\mathrm{kg} / \mathrm{m}^{3}$ and 0.2 , respectively. The nacelle has a Young's modulus $500 \mathrm{GPa}$, Poisson's ratio 0.2, and density $741 \mathrm{~kg} / \mathrm{m}^{3}$ to produce a realistically stiff structure with a mass of 82 metric tons. Given these design characteristics, the combined tower and nacelle structure has a mass of approximately 1,662 metric tons.

For the NREL $5 \mathrm{MW}$ rotor design, we use the geometry definition provided in [156] to generate an initial blade model using the Grasshopper algorithmic modeling plugin for Rhinoceros. We then scale the blade by a factor appropriate to achieve a $108 \mathrm{~m}$ rotor and convert the model to a T-spline geometry description. Three such blades are then attached to a hub with a precone angle of 2.5 degrees to produce the fi- nal rotor model. A simplified blade structural model is considered in this work. Internal shear webs are not modeled, and an isotropic material with an assumed thickness distribution is used (more details can be found in [87]). The Young's modulus and Poisson's ratio are set to $55.2 \mathrm{GPa}$ and 0.2 , respectively. The density is set to $2500 \mathrm{~kg} / \mathrm{m}^{3}$. Material properties and shell thickness distribution are selected such that the rotor has a mass of $60,000 \mathrm{~kg}$, and such that the blade undergoes reasonable deflection and has a natural frequency of $0.705 \mathrm{~Hz}$. This frequency was calculated using a simple proportional scaling law [157] applied to the original NREL 5 MW blade natural frequency of $0.870 \mathrm{~Hz}$. Figure 20 shows the rotor model, where the T-spline mesh 




Fig. 20: T-spline mesh of the rotor surface.
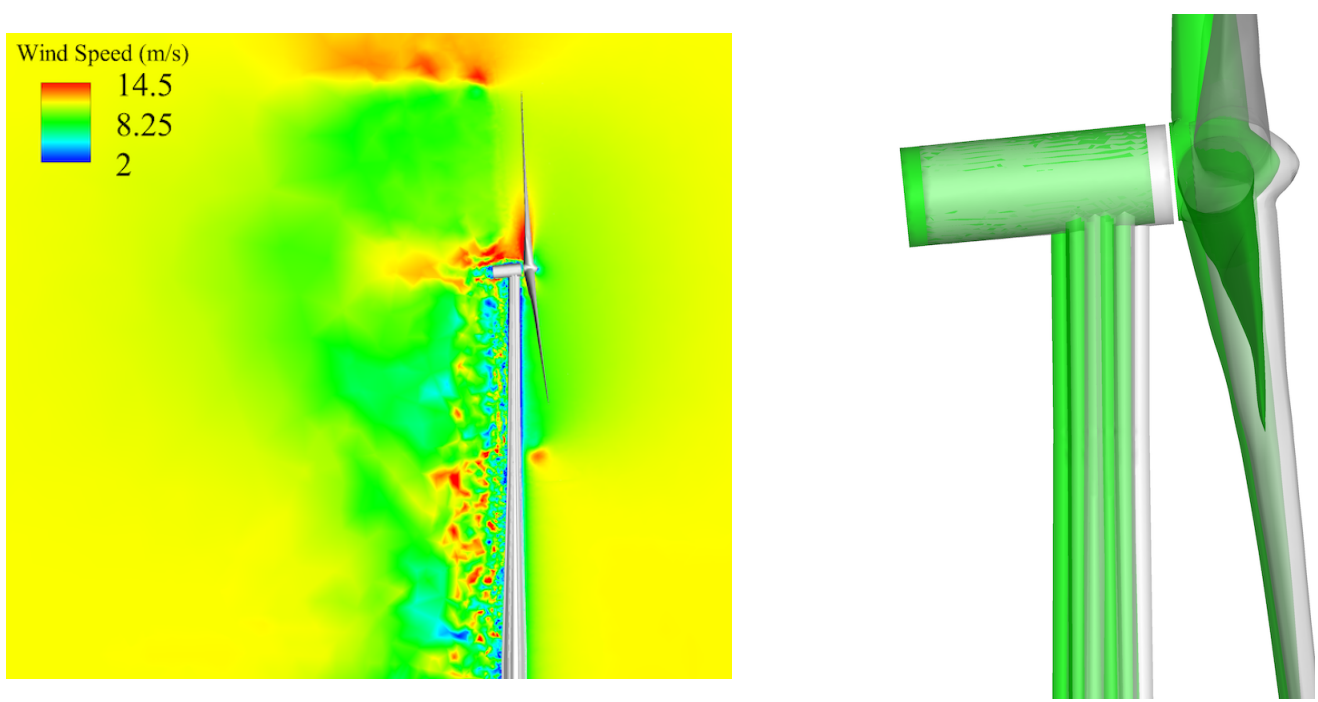

Fig. 21: Air speed contours at a planar cut (left) and wind-turbine deflected shape (right). The undeformed structure is shown in gray and the deformed structure is shown in light green.

consists of $23,244 C^{1}$-continuous cubic elements and 25,151 control points.



Fig. 22: Penalty coupling error as a function of time.

\subsection{Results}

The FSI simulation is performed at the rated wind speed of $11.4 \mathrm{~m} / \mathrm{s}$. Figure 21 shows the flow visualization of the full wind turbine con- figuration, and the deflection of the tower and blades.

The figure clearly demonstrates that the rotor and tower displacements are coupled while the rotor is spinning. To assess the penalty-coupling error $E_{\text {int }}$ we define it as

$$
\begin{aligned}
& E_{\text {int }} \\
& \equiv \frac{\int_{\Gamma_{1}} \int_{\Gamma_{2}}\left(\left\|\mathbf{x}_{1}-\mathbf{x}_{2}\right\|-\left\|\mathbf{X}_{1}^{r}-\mathbf{X}_{2}^{r}\right\|\right)^{2} \mathrm{~d} \Gamma_{2} \mathrm{~d} \Gamma_{1}}{\int_{\Gamma_{1}} \int_{\Gamma_{2}}\left\|\mathbf{X}_{1}^{r}-\mathbf{X}_{2}^{r}\right\|^{2} \mathrm{~d} \Gamma_{2} \mathrm{~d} \Gamma_{1}},
\end{aligned}
$$

and plot it a function of time in Figure 22. The figure clearly shows that the coupling error, defined as a relative, dimensionless quantity, is very small.

\section{Concluding remarks}

We have described how the challenges encountered in computational analysis of wind turbines 
and turbomachinery are being addressed by the ALE-VMS and ST-VMS methods and isogeometric discretization. The computational challenges include turbulent rotational flows, complex geometries, MBI, such as the rotor motion, and the FSI, such as the FSI between the wind turbine blade and the air. The ALE-VMS and ST-VMS serve as the core computational methods. They are supplemented with special methods like the ST-ALE and ST-SI, weak enforcement of the no-slip boundary conditions, and ST-IGA with NURBS basis functions in time. We described the core methods and some of the special methods. We presented, as examples of challenging computations performed, computational analysis of a HAWT, a VAWT and flowdriven string dynamics in pumps. The examples demonstrate the power and scope of the core and special methods in computational analysis of wind turbines and turbomachinery.

\section{Acknowledgment}

This work was supported (second author) in part by Grant-in-Aid for Challenging Exploratory Research 16K13779 from Japan Society for the Promotion of Science; Grant-in-Aid for Scientific Research (S) 26220002 from the Ministry of Education, Culture, Sports, Science and Technology of Japan (MEXT); Council for Science, Technology and Innovation (CSTI), CrossMinisterial Strategic Innovation Promotion Program (SIP), "Innovative Combustion Technology" (Funding agency: JST); and Rice-Waseda research agreement. It was also supported in part by Grant-in-Aid for Early-Career Scientists 19K20287 (fifth author). The mathematical model and computational method parts of the work were also supported (third author) in part by ARO Grant W911NF-17-1-0046, ARO DURIP Grant W911NF-18-1-0234, and Top Global University Project of Waseda University. The first author was partially supported by NSF Grant 1854436, and the fourth author was partially supported by NIH/NHLBI Grants R01HL129077 and R01HL142504.

\section{References}

[1] Takizawa, K., Tezduyar, T. E., Mochizuki, H., Hattori, H., Mei, S., Pan, L., \& Montel, K. (2015). Space-time VMS method for flow computations with slip interfaces (ST-SI). Mathematical Models and Methods in Applied Sciences, 25, 2377-2406.

[2] Bazilevs, Y., Korobenko, A., Deng, X., \& Yan, J. (2016). FSI modeling for fatigue-damage prediction in full-scale wind-turbine blades. Journal of Applied Mechanics, 83(6), 061010.

[3] Kanai, T., Takizawa, K., Tezduyar, T. E., Komiya, K., Kaneko, M., Hirota, K., Nohmi, M., Tsuneda, T., Kawai, M., \& Isono, M. (2019). Methods for Computation of Flow-Driven String Dynamics in a Pump and Residence Time. Mathematical Models and Methods in Applied Sciences, $29,839-870$.

[4] Korobenko, A., Bazilevs, Y., Takizawa, K., \& Tezduyar, T. E. (2019). Computer modeling of wind turbines: 1. ALE-VMS and ST-VMS aerodynamic and FSI analysis. Archives of Computational Methods in Engineering, 26, 1059-1099.

[5] Bazilevs, Y., Calo, V. M., Hughes, T. J. R., \& Zhang, Y. (2008). Isogeometric fluid-structure interaction: theory, algorithms, and computations. Computational Mechanics, 43, 3-37.

[6] Takizawa, K., \& Tezduyar, T. E. (2011). Multiscale Space-Time Fluid-Structure Interaction Techniques. Computational Mechanics, 48, 247-267.

[7] Takizawa, K., Tezduyar, T. E., Kuraishi, T., Tabata, S., \& Takagi, H. (2016). Computational thermo-fluid analysis of a disk brake. Computational Mechanics, 57, 965977.

[8] Takizawa, K., Henicke, B., Puntel, A., Spielman, T., \& Tezduyar, T. E. (2012). Space-time computational techniques for the aerodynamics of flapping wings. Journal of Applied Mechanics, 79, 010903. 
[9] Takizawa, K., Tezduyar, T. E., Otoguro, Y., Terahara, T., Kuraishi, T., \& Hattori, H. (2017). Turbocharger Flow Computations with the Space-Time Isogeometric Analysis (ST-IGA). Computers \& Fluids, 142, 15-20.

[10] Otoguro, Y., Takizawa, K., \& Tezduyar, T. E. (2017). Space-time VMS computational flow analysis with isogeometric discretization and a general-purpose NURBS mesh generation method. Computers \& Fluids, 158, 189-200.

[11] Otoguro, Y., Takizawa, K., \& Tezduyar, T. E. (2018). A General-Purpose NURBS Mesh Generation Method for Complex Geometries. In: Tezduyar, T. E. (Ed.), Frontiers in Computational FluidStructure Interaction and Flow Simulation: Research from Lead Investigators under Forty - 2018, Modeling and Simulation in Science, Engineering and Technology. Springer, 399-434.

[12] Takizawa, K., \& Tezduyar, T. E. (2012). Computational Methods for Parachute Fluid-Structure Interactions. Archives of Computational Methods in Engineering, 19, 125-169.

[13] Bazilevs, Y., \& Hughes, T. J. R. (2007). Weak imposition of Dirichlet boundary conditions in fluid mechanics. Computers and Fluids, 36, 12-26.

[14] Bazilevs, Y., Michler, C., Calo, V. M., \& Hughes, T. J. R. (2010). Isogeometric variational multiscale modeling of wall-bounded turbulent flows with weakly enforced boundary conditions on unstretched meshes. Computer Methods in Applied Mechanics and Engineering, 199, 780-790.

[15] Hsu, M.-C., Akkerman, I., \& Bazilevs, Y. (2012). Wind turbine aerodynamics using ALE-VMS: Validation and role of weakly enforced boundary conditions. Computational Mechanics, 50, 499-511.

[16] Bazilevs, Y., \& Hughes, T. J. R. (2008). NURBS-based isogeometric analysis for the computation of flows about rotating components. Computational Mechanics, $43,143-150$.

[17] Hsu, M.-C., \& Bazilevs, Y. (2012). Fluidstructure interaction modeling of wind turbines: simulating the full machine. Computational Mechanics, 50, 821-833.

[18] Bazilevs, Y., Takizawa, K., Tezduyar, T. E., Hsu, M.-C., Otoguro, Y., Mochizuki, H., \& Wu, M. C. H. (2020). ALE and Space-Time Variational Multiscale Isogeometric Analysis of Wind Turbines and Turbomachinery. In: Grama, A., \& Sameh, A. (Eds.), Parallel Algorithms in Computational Science and Engineering, Modeling and Simulation in Science, Engineering and Technology. Springer.

[19] Bazilevs, Y., Takizawa, K., \& Tezduyar, T. E. (February 2013). Computational Fluid-Structure Interaction: Methods and Applications. Wiley.

[20] Tezduyar, T. E. (1992). Stabilized Finite Element Formulations for Incompressible Flow Computations. Advances in Applied Mechanics, 28, 1-44.

[21] Tezduyar, T. E. (2003). Computation of Moving Boundaries and Interfaces and Stabilization Parameters. International Journal for Numerical Methods in Fluids, $43,555-575$.

[22] Tezduyar, T. E., \& Sathe, S. (2007). Modeling of Fluid-Structure Interactions with the Space-Time Finite Elements: Solution Techniques. International Journal for $\mathrm{Nu}^{-}$ merical Methods in Fluids, 54, 855-900.

[23] Brooks, A. N., \& Hughes, T. J. R. (1982). Streamline Upwind/PetrovGalerkin Formulations for Convection Dominated Flows with Particular Emphasis on the Incompressible Navier-Stokes Equations. Computer Methods in Applied Mechanics and Engineering, 32, 199-259.

[24] Hughes, T. J. R. (1995). Multiscale Phenomena: Green's Functions, The 
Dirichlet-to-Neumann Formulation, Subgrid Scale Models, Bubbles, and the Origins of Stabilized Methods. Computer Methods in Applied Mechanics and Engineering, 127, 387-401.

[25] Hughes, T. J. R., Oberai, A. A., \& Mazzei, L. (2001). Large Eddy Simulation of Turbulent Channel Flows by the Variational Multiscale Method. Physics of Fluids, 13, 1784-1799.

[26] Bazilevs, Y., Calo, V. M., Cottrell, J. A., Hughes, T. J. R., Reali, A., \& Scovazzi, G. (2007). Variational multiscale residualbased turbulence modeling for large eddy simulation of incompressible flows. Computer Methods in Applied Mechanics and Engineering, 197, 173-201.

[27] Bazilevs, Y., \& Akkerman, I. (2010). Large eddy simulation of turbulent Taylor-Couette flow using isogeometric analysis and the residual-based variational multiscale method. Journal of Computational Physics, 229, 3402-3414.

[28] Takizawa, K., \& Tezduyar, T. E. (2012). Space-Time Fluid-Structure Interaction Methods. Mathematical Models and Methods in Applied Sciences, 22(supp02), 1230001.

[29] Tezduyar, T. E., \& Takizawa, K. (2019). Space-time computations in practical engineering applications: A summary of the 25-year history. Computational Mechanics, 63, 747-753.

[30] Takizawa, K., Fritze, M., Montes, D., Spielman, T., \& Tezduyar, T. E. (2012). Fluid-structure interaction modeling of ringsail parachutes with disreefing and modified geometric porosity. Computational Mechanics, 50, 835-854.

[31] Takizawa, K., Tezduyar, T. E., Boben, J., Kostov, N., Boswell, C., \& Buscher, A. (2013). Fluid-structure interaction modeling of clusters of spacecraft parachutes with modified geometric porosity. Computational Mechanics, 52, 1351-1364.
[32] Takizawa, K., Tezduyar, T. E., Boswell, C., Tsutsui, Y., \& Montel, K. (2015). Special Methods for Aerodynamic-Moment Calculations from Parachute FSI Modeling. Computational Mechanics, 55, 10591069 .

[33] Takizawa, K., Montes, D., Fritze, M., McIntyre, S., Boben, J., \& Tezduyar, T. E. (2013). Methods for FSI modeling of spacecraft parachute dynamics and cover separation. Mathematical Models and Methods in Applied Sciences, 23, 307338 .

[34] Takizawa, K., Tezduyar, T. E., Boswell, C., Kolesar, R., \& Montel, K. (2014). FSI Modeling of the Reefed Stages and Disreefing of the Orion Spacecraft Parachutes. Computational Mechanics, 54, 1203-1220.

[35] Takizawa, K., Tezduyar, T. E., Kolesar, R., Boswell, C., Kanai, T., \& Montel, K. (2014). Multiscale Methods for Gore Curvature Calculations from FSI Modeling of Spacecraft Parachutes. Computational Mechanics, 54, 1461-1476.

[36] Takizawa, K., Tezduyar, T. E., \& Kolesar, R. (2015). FSI Modeling of the Orion Spacecraft Drogue Parachutes. Computational Mechanics, 55, 1167-1179.

[37] Bazilevs, Y., Hsu, M.-C., Akkerman, I., Wright, S., Takizawa, K., Henicke, B., Spielman, T., \& Tezduyar, T. E. (2011). 3D Simulation of Wind Turbine Rotors at Full Scale. Part I: Geometry Modeling and Aerodynamics. International Journal for Numerical Methods in Fluids, 65, 207235 .

[38] Takizawa, K., Henicke, B., Tezduyar, T. E., Hsu, M.-C., \& Bazilevs, Y. (2011). Stabilized Space-Time Computation of Wind-Turbine Rotor Aerodynamics. Computational Mechanics, 48, 333-344.

[39] Takizawa, K., Henicke, B., Montes, D., Tezduyar, T. E., Hsu, M.-C., \& Bazilevs, Y. (2011). Numerical-Performance Studies for the Stabilized Space-Time Computation of Wind-Turbine Rotor Aerodynam- 
ics. Computational Mechanics, 48, 647657.

[40] Takizawa, K., Tezduyar, T. E., McIntyre, S., Kostov, N., Kolesar, R., \& Habluetzel, C. (2014). Space-time VMS computation of wind-turbine rotor and tower aerodynamics. Computational Mechanics, 53, 115.

[41] Takizawa, K., Bazilevs, Y., Tezduyar, T. E., Hsu, M.-C., Øiseth, O., Mathisen, K. M., Kostov, N., \& McIntyre, S. (2014). Engineering Analysis and Design with ALE-VMS and Space-Time Methods. Archives of Computational Methods in Engineering, 21, 481-508.

[42] Takizawa, K. (2014). Computational Engineering Analysis with the NewGeneration Space-Time Methods. Computational Mechanics, 54, 193-211.

[43] Bazilevs, Y., Takizawa, K., Tezduyar, T. E., Hsu, M.-C., Kostov, N., \& McIntyre, S. (2014). Aerodynamic and FSI Analysis of Wind Turbines with the ALEVMS and ST-VMS Methods. Archives of Computational Methods in Engineering, $21,359-398$.

[44] Korobenko, A., Bazilevs, Y., Takizawa, K., \& Tezduyar, T. E. (2018). Recent Advances in ALE-VMS and ST-VMS Computational Aerodynamic and FSI Analysis of Wind Turbines. In: Tezduyar, T. E. (Ed.), Frontiers in Computational FluidStructure Interaction and Flow Simulation: Research from Lead Investigators under Forty - 2018, Modeling and Simulation in Science, Engineering and Technology. Springer, 253-336.

[45] Takizawa, K., Henicke, B., Puntel, A., Kostov, N., \& Tezduyar, T. E. (2012). Space-Time Techniques for Computational Aerodynamics Modeling of Flapping Wings of an Actual Locust. Computational Mechanics, 50, 743-760.

[46] Takizawa, K., Henicke, B., Puntel, A., Kostov, N., \& Tezduyar, T. E. (2013). Computer Modeling Techniques for Flapping-Wing Aerodynamics of a Locust. Computers \& Fluids, 85, 125-134.

[47] Takizawa, K., Kostov, N., Puntel, A., Henicke, B., \& Tezduyar, T. E. (2012). Space-time computational analysis of bioinspired flapping-wing aerodynamics of a micro aerial vehicle. Computational Mechanics, 50, 761-778.

[48] Takizawa, K., Tezduyar, T. E., \& Kostov, N. (2014). Sequentially-coupled spacetime FSI analysis of bio-inspired flappingwing aerodynamics of an MAV. Computational Mechanics, 54, 213-233.

[49] Takizawa, K., Tezduyar, T. E., Buscher, A., \& Asada, S. (2014). Space-Time Interface-Tracking with Topology Change (ST-TC). Computational Mechanics, 54, 955-971.

[50] Takizawa, K., Tezduyar, T. E., \& Buscher, A. (2015). Space-Time Computational Analysis of MAV Flapping-Wing Aerodynamics with Wing Clapping. Computational Mechanics, 55, 1131-1141.

[51] Takizawa, K., Bazilevs, Y., Tezduyar, T. E., Long, C. C., Marsden, A. L., \& Schjodt, K. (2014). ST and ALE-VMS Methods for Patient-Specific Cardiovascular Fluid Mechanics Modeling. Mathematical Models and Methods in Applied Sciences, 24, 2437-2486.

[52] Takizawa, K., Schjodt, K., Puntel, A., Kostov, N., \& Tezduyar, T. E. (2012). Patient-specific computer modeling of blood flow in cerebral arteries with aneurysm and stent. Computational Mechanics, 50, 675-686.

[53] Takizawa, K., Schjodt, K., Puntel, A., Kostov, N., \& Tezduyar, T. E. (2013). Patient-Specific Computational Analysis of the Influence of a Stent on the Unsteady Flow in Cerebral Aneurysms. Computational Mechanics, 51, 1061-1073.

[54] Suito, H., Takizawa, K., Huynh, V. Q. H., Sze, D., \& Ueda, T. (2014). FSI analysis of the blood flow and geometrical characteristics in the thoracic aorta. Computational Mechanics, 54, 1035-1045. 
[55] Suito, H., Takizawa, K., Huynh, V. Q. H., Sze, D., Ueda, T., \& Tezduyar, T. E. (2016). A geometrical-characteristics study in patient-specific FSI analysis of blood flow in the thoracic aorta. In: Bazilevs, Y., \& Takizawa, K. (Eds.), Advances in Computational Fluid-Structure Interaction and Flow Simulation: New Methods and Challenging Computations, Modeling and Simulation in Science, Engineering and Technology. Springer, 379386.

[56] Takizawa, K., Tezduyar, T. E., Uchikawa, H., Terahara, T., Sasaki, T., Shiozaki, K., Yoshida, A., Komiya, K., \& Inoue, G. (2018). Aorta Flow Analysis and Heart Valve Flow and Structure Analysis. In: Tezduyar, T. E. (Ed.), Frontiers in Computational Fluid-Structure Interaction and Flow Simulation: Research from Lead Investigators under Forty 2018, Modeling and Simulation in Science, Engineering and Technology. Springer, 2989.

[57] Takizawa, K., Tezduyar, T. E., Uchikawa, H., Terahara, T., Sasaki, T., \& Yoshida, A. (2019). Mesh refinement influence and cardiac-cycle flow periodicity in aorta flow analysis with isogeometric discretization. Computers \& Fluids, 179, 790-798.

[58] Takizawa, K., Bazilevs, Y., Tezduyar, T. E., \& Hsu, M.-C. (2019). Computational Cardiovascular Flow Analysis with the Variational Multiscale Methods. Journal of Advanced Engineering and Computation, 3, 366-405.

[59] Takizawa, K., Tezduyar, T. E., Buscher, A., \& Asada, S. (2014). Space-Time Fluid Mechanics Computation of Heart Valve Models. Computational Mechanics, 54, 973-986.

[60] Takizawa, K., \& Tezduyar, T. E. (2016). New directions in space-time computational methods. In: Bazilevs, Y., \& Takizawa, K. (Eds.), Advances in Computational Fluid-Structure Interaction and Flow Simulation: New Methods and Challenging Computations, Modeling and Sim- ulation in Science, Engineering and Technology. Springer, 159-178.

[61] Takizawa, K., Tezduyar, T. E., Terahara, T., \& Sasaki, T. (2018). Heart valve flow computation with the Space-Time Slip Interface Topology Change (ST-SITC) method and Isogeometric Analysis (IGA). In: Wriggers, P., \& Lenarz, T. (Eds.), Biomedical Technology: Modeling, Experiments and Simulation, Lecture Notes in Applied and Computational Mechanics. Springer, 77-99.

[62] Takizawa, K., Tezduyar, T. E., Terahara, T., \& Sasaki, T. (2017). Heart valve flow computation with the integrated Space-Time VMS, Slip Interface, Topology Change and Isogeometric Discretization methods. Computers \& Fluids, 158, 176-188.

[63] Terahara, T., Takizawa, K., Tezduyar, T. E., Bazilevs, Y., \& Hsu, M.-C. (2020). Heart Valve Isogeometric SequentiallyCoupled FSI Analysis with the SpaceTime Topology Change Method. Computational Mechanics, published online, DOI: $10.1007 / \mathrm{s} 00466-019-01813-0$.

[64] Terahara, T., Takizawa, K., Tezduyar, T. E., Tsushima, A., \& Shiozaki, K. (2020). Ventricle-valve-aorta flow analysis with the Space-Time Isogeometric Discretization and Topology Change. Computational Mechanics, to appear, DOI: 10.1007/s00466-020-01822-4.

[65] Yu, Y., Zhang, Y. J., Takizawa, K., Tezduyar, T. E., \& Sasaki, T. (2020). Anatomically Realistic Lumen Motion Representation in Patient-Specific Space-Time Isogeometric Flow Analysis of Coronary Arteries with Time-Dependent Medical-Image Data. Computational Mechanics, 65, 395404.

[66] Takizawa, K., Montes, D., McIntyre, S., \& Tezduyar, T. E. (2013). Space-Time VMS Methods for Modeling of Incompressible Flows at High Reynolds Numbers. Mathematical Models and Methods in Applied Sciences, 23, 223-248. 
[67] Takizawa, K., Tezduyar, T. E., \& Kuraishi, T. (2015). Multiscale ST Methods for Thermo-Fluid Analysis of a Ground Vehicle and its Tires. Mathematical Models and Methods in Applied Sciences, 25, $2227-2255$.

[68] Takizawa, K., Tezduyar, T. E., \& Hattori, H. (2017). Computational Analysis of Flow-Driven String Dynamics in Turbomachinery. Computers \& Fluids, 142, 109-117.

[69] Komiya, K., Kanai, T., Otoguro, Y., Kaneko, M., Hirota, K., Zhang, Y., Takizawa, K., Tezduyar, T. E., Nohmi, M., Tsuneda, T., Kawai, M., \& Isono, M. (2019). Computational analysis of flowdriven string dynamics in a pump and residence time calculation. IOP conference series earth and environmental science, 240 , 062014 .

[70] Otoguro, Y., Takizawa, K., Tezduyar, T. E., Nagaoka, K., \& Mei, S. (2019). Turbocharger turbine and exhaust manifold flow computation with the SpaceTime Variational Multiscale Method and Isogeometric Analysis. Computers \& Fluids, $179,764-776$.

[71] Otoguro, Y., Takizawa, K., Tezduyar, T. E., Nagaoka, K., Avsar, R., \& Zhang, Y. (2019). Space-Time VMS Flow Analysis of a Turbocharger Turbine with Isogeometric Discretization: Computations with Time-Dependent and Steady-Inflow Representations of the Intake/Exhaust Cycle. Computational Mechanics, 64, 1403-1419.

[72] Takizawa, K., Tezduyar, T. E., Asada, S., \& Kuraishi, T. (2016). Space-Time Method for Flow Computations with Slip Interfaces and Topology Changes (ST-SITC). Computers \& Fluids, 141, 124-134.

[73] Kuraishi, T., Takizawa, K., \& Tezduyar, T. E. (2018). Space-Time Computational Analysis of Tire Aerodynamics with Actual Geometry, Road Contact and Tire Deformation. In: Tezduyar, T. E. (Ed.), Frontiers in Computational FluidStructure Interaction and Flow Simulation: Research from Lead Investigators under Forty - 2018, Modeling and Simulation in Science, Engineering and Technology. Springer, 337-376.

[74] Kuraishi, T., Takizawa, K., \& Tezduyar, T. E. (2019). Tire Aerodynamics with Actual Tire Geometry, Road Contact and Tire Deformation. Computational Mechanics, 63, 1165-1185.

[75] Kuraishi, T., Takizawa, K., \& Tezduyar, T. E. (2019). Space-Time Computational Analysis of Tire Aerodynamics with Actual Geometry, Road Contact, Tire Deformation, Road Roughness and Fluid Film. Computational Mechanics, 64, 1699-1718.

[76] Kuraishi, T., Takizawa, K., \& Tezduyar, T. E. (2019). Space-Time Isogeometric Flow Analysis with Built-in ReynoldsEquation Limit. Mathematical Models and Methods in Applied Sciences, 29, 871904.

[77] Takizawa, K., Tezduyar, T. E., \& Terahara, T. (2016). Ram-Air Parachute Structural and Fluid Mechanics Computations with the Space-Time Isogeometric Analysis (ST-IGA). Computers \& Fluids, 141, 191-200.

[78] Takizawa, K., Tezduyar, T. E., \& Kanai, T. (2017). Porosity models and computational methods for compressible-flow aerodynamics of parachutes with geometric porosity. Mathematical Models and Methods in Applied Sciences, 27, 771-806.

[79] Kanai, T., Takizawa, K., Tezduyar, T. E., Tanaka, T., \& Hartmann, A. (2019). Compressible-Flow Geometric-Porosity Modeling and Spacecraft Parachute Computation with Isogeometric Discretization. Computational Mechanics, 63, 301-321.

[80] Takizawa, K., Bazilevs, Y., \& Tezduyar, T. E. (2012). Space-Time and ALE-VMS Techniques for Patient-Specific Cardiovascular Fluid-Structure Interaction Modeling. Archives of Computational Methods in Engineering, 19, 171-225.

[81] Bazilevs, Y., Hsu, M.-C., Takizawa, K., \& Tezduyar, T. E. (2012). ALE-VMS and 
ST-VMS Methods for Computer Modeling of Wind-Turbine Rotor Aerodynamics and Fluid-Structure Interaction. Mathematical Models and Methods in Applied Sciences, 22(supp02), 1230002.

[82] Bazilevs, Y., Takizawa, K., \& Tezduyar, T. E. (2013). Challenges and Directions in Computational Fluid-Structure Interaction. Mathematical Models and Methods in Applied Sciences, 23, 215-221.

[83] Bazilevs, Y., Takizawa, K., \& Tezduyar, T. E. (2015). New Directions and Challenging Computations in Fluid Dynamics Modeling with Stabilized and Multiscale Methods. Mathematical Models and Methods in Applied Sciences, 25, 22172226 .

[84] Bazilevs, Y., Takizawa, K., \& Tezduyar, T. E. (2019). Computational Analysis Methods for Complex Unsteady Flow Problems. Mathematical Models and Methods in Applied Sciences, 29, 825-838.

[85] Hughes, T. J. R., Liu, W. K., \& Zimmermann, T. K. (1981). Lagrangian-Eulerian finite element formulation for incompressible viscous flows. Computer Methods in Applied Mechanics and Engineering, 29, 329-349.

[86] Kalro, V., \& Tezduyar, T. E. (2000). A Parallel 3D Computational Method for Fluid-Structure Interactions in Parachute Systems. Computer Methods in Applied Mechanics and Engineering, 190, 321-332.

[87] Bazilevs, Y., Hsu, M.-C., Kiendl, J., Wüchner, R., \& Bletzinger, K.-U. (2011). 3D simulation of wind turbine rotors at full scale. Part II: Fluid-structure interaction modeling with composite blades. International Journal for Numerical Methods in Fluids, 65, 236-253.

[88] Hsu, M.-C., Akkerman, I., \& Bazilevs, Y. (2011). High-performance computing of wind turbine aerodynamics using isogeometric analysis. Computers and Fluids, $49,93-100$.
[89] Bazilevs, Y., Hsu, M.-C., \& Scott, M. A. (2012). Isogeometric Fluid-Structure Interaction Analysis with Emphasis on NonMatching Discretizations, and with Application to Wind Turbines. Computer Methods in Applied Mechanics and Engineering, 249-252, 28-41.

[90] Hsu, M.-C., Akkerman, I., \& Bazilevs, Y. (2014). Finite element simulation of wind turbine aerodynamics: Validation study using NREL Phase VI experiment. Wind Energy, 17, 461-481.

[91] Korobenko, A., Hsu, M.-C., Akkerman, I., Tippmann, J., \& Bazilevs, Y. (2013). Structural mechanics modeling and FSI simulation of wind turbines. Mathematical Models and Methods in Applied Sciences, 23, 249-272.

[92] Bazilevs, Y., Korobenko, A., Deng, X., \& Yan, J. (2015). Novel structural modeling and mesh moving techniques for advanced FSI simulation of wind turbines. International Journal for Numerical Methods in Engineering, 102, 766-783.

[93] Korobenko, A., Yan, J., Gohari, S. M. I., Sarkar, S., \& Bazilevs, Y. (2017). FSI simulation of two back-to-back wind turbines in atmospheric boundary layer flow. Computers \& Fluids, 158, 167-175.

[94] Korobenko, A., Hsu, M.-C., Akkerman, I., \& Bazilevs, Y. (2013). Aerodynamic simulation of vertical-axis wind turbines. Journal of Applied Mechanics, 81, 021011.

[95] Bazilevs, Y., Korobenko, A., Deng, X., Yan, J., Kinzel, M., \& Dabiri, J. O. (2014). FSI modeling of vertical-axis wind turbines. Journal of Applied Mechanics, $81,081006$.

[96] Yan, J., Korobenko, A., Deng, X., \& Bazilevs, Y. (2016). Computational freesurface fluid-structure interaction with application to floating offshore wind turbines. Computers and Fluids, 141, 155174.

[97] Bazilevs, Y., Korobenko, A., Yan, J., Pal, A., Gohari, S. M. I., \& Sarkar, S. (2015). 
ALE-VMS Formulation for Stratified Turbulent Incompressible Flows with Applications. Mathematical Models and Methods in Applied Sciences, 25, 2349-2375.

[98] Bazilevs, Y., Calo, V. M., Zhang, Y., \& Hughes, T. J. R. (2006). Isogeometric fluid-structure interaction analysis with applications to arterial blood flow. Computational Mechanics, 38, 310-322.

[99] Bazilevs, Y., Gohean, J. R., Hughes, T. J. R., Moser, R. D., \& Zhang, Y. (2009). Patient-specific isogeometric fluidstructure interaction analysis of thoracic aortic blood flow due to implantation of the Jarvik 2000 left ventricular assist device. Computer Methods in Applied Mechanics and Engineering, 198, 3534-3550.

[100] Bazilevs, Y., Hsu, M.-C., Benson, D., Sankaran, S., \& Marsden, A. (2009). Computational Fluid-Structure Interaction: Methods and Application to a Total Cavopulmonary Connection. Computational Mechanics, 45, 77-89.

[101] Bazilevs, Y., Hsu, M.-C., Zhang, Y., Wang, W., Liang, X., Kvamsdal, T., Brekken, R., \& Isaksen, J. (2010). A Fully-Coupled Fluid-Structure Interaction Simulation of Cerebral Aneurysms. Computational Mechanics, 46, 3-16.

[102] Bazilevs, Y., Hsu, M.-C., Zhang, Y., Wang, W., Kvamsdal, T., Hentschel, S., \& Isaksen, J. (2010). Computational FluidStructure Interaction: Methods and Application to Cerebral Aneurysms. Biomechanics and Modeling in Mechanobiology, 9, 481-498.

[103] Hsu, M.-C., \& Bazilevs, Y. (2011). Blood vessel tissue prestress modeling for vascular fluid-structure interaction simulations. Finite Elements in Analysis and Design, 47, 593-599.

[104] Long, C. C., Marsden, A. L., \& Bazilevs, Y. (2013). Fluid-structure interaction simulation of pulsatile ventricular assist devices. Computational Mechanics, 52, 971-981.
[105] Long, C. C., Esmaily-Moghadam, M., Marsden, A. L., \& Bazilevs, Y. (2014). Computation of residence time in the simulation of pulsatile ventricular assist devices. Computational Mechanics, 54, 911919.

[106] Long, C. C., Marsden, A. L., \& Bazilevs, Y. (2014). Shape optimization of pulsatile ventricular assist devices using FSI to minimize thrombotic risk. Computational Mechanics, 54, 921-932.

[107] Hsu, M.-C., Kamensky, D., Bazilevs, Y., Sacks, M. S., \& Hughes, T. J. R. (2014). Fluid-structure interaction analysis of bioprosthetic heart valves: significance of arterial wall deformation. Computational Mechanics, 54, 1055-1071.

[108] Hsu, M.-C., Kamensky, D., Xu, F., Kiendl, J., Wang, C., Wu, M. C. H., Mineroff, J., Reali, A., Bazilevs, Y., \& Sacks, M. S. (2015). Dynamic and fluid-structure interaction simulations of bioprosthetic heart valves using parametric design with $\mathrm{T}$ splines and Fung-type material models. Computational Mechanics, 55, 1211-1225.

[109] Kamensky, D., Hsu, M.-C., Schillinger, D., Evans, J. A., Aggarwal, A., Bazilevs, Y., Sacks, M. S., \& Hughes, T. J. R. (2015). An immersogeometric variational framework for fluid-structure interaction: Application to bioprosthetic heart valves. Computer Methods in Applied Mechanics and Engineering, 284, 1005-1053.

[110] Akkerman, I., Bazilevs, Y., Benson, D. J., Farthing, M. W., \& Kees, C. E. (2012). Free-Surface Flow and Fluid-Object Interaction Modeling with Emphasis on Ship Hydrodynamics. Journal of Applied Mechanics, 79, 010905.

[111] Akkerman, I., Dunaway, J., Kvandal, J., Spinks, J., \& Bazilevs, Y. (2012). Toward free-surface modeling of planing vessels: simulation of the Fridsma hull using ALE-VMS. Computational Mechanics, 50, 719-727. 
[112] Wang, C., Wu, M. C. H., Xu, F., Hsu, M.-C., \& Bazilevs, Y. (2017). Modeling of a hydraulic arresting gear using fluid-structure interaction and isogeometric analysis. Computers and Fluids, 142, 3-14.

[113] Wu, M. C. H., Kamensky, D., Wang, C., Herrema, A. J., Xu, F., Pigazzini, M. S., Verma, A., Marsden, A. L., Bazilevs, Y., \& Hsu, M.-C. (2017). Optimizing fluidstructure interaction systems with immersogeometric analysis and surrogate modeling: Application to a hydraulic arresting gear. Computer Methods in Applied Mechanics and Engineering, 316, 668-693.

[114] Yan, J., Deng, X., Korobenko, A., \& Bazilevs, Y. (2017). Free-surface flow modeling and simulation of horizontal-axis tidal-stream turbines. Computers and Fluids, 158, 157-166.

[115] Castorrini, A., Corsini, A., Rispoli, F., Takizawa, K., \& Tezduyar, T. E. (2019). A stabilized ALE method for computational fluid-structure interaction analysis of passive morphing in turbomachinery. Mathematical Models and Methods in Applied Sciences, 29, 967-994.

[116] Augier, B., Yan, J., Korobenko, A., Czarnowski, J., Ketterman, G., \& Bazilevs, Y. (2015). Experimental and numerical FSI study of compliant hydrofoils. Computational Mechanics, 55, 1079-1090.

[117] Yan, J., Augier, B., Korobenko, A., Czarnowski, J., Ketterman, G., \& Bazilevs, Y. (2016). FSI modeling of a propulsion system based on compliant hydrofoils in a tandem configuration. Computers and Fluids, 141, 201-211.

[118] Helgedagsrud, T. A., Bazilevs, Y., Mathisen, K. M., \& Oiseth, O. A. Computational and experimental investigation of free vibration and flutter of bridge decks. Computational Mechanics. Published online.

[119] Helgedagsrud, T. A., Bazilevs, Y., Korobenko, A., Mathisen, K. M., \& Oiseth,
O. A. Using ALE-VMS to compute aerodynamic derivatives of bridge sections. Computers and Fluids. Published online.

[120] Helgedagsrud, T. A., Akkerman, I., Bazilevs, Y., Mathisen, K. M., \& Oiseth, O. A. (2019). Isogeometric modeling and experimental investigation of movingdomain bridge aerodynamics. ASCE Journal of Engineering Mechanics, 145, 04019026.

[121] Kamensky, D., Evans, J. A., Hsu, M.C., \& Bazilevs, Y. (2017). Projectionbased stabilization of interface Lagrange multipliers in immersogeometric fluid-thin structure interaction analysis, with application to heart valve modeling. Computers and Mathematics with Applications, 74, 2068-2088.

[122] Yu, Y., Kamensky, D., Hsu, M.-C., Lu, X. Y., Bazilevs, Y., \& Hughes, T. J. R. (2018). Error estimates for projection-based dynamic augmented Lagrangian boundary condition enforcement, with application to fluid-structure interaction. Mathematical Models and Methods in Applied Science, 28, 2457-2509.

[123] Tezduyar, T. E., Takizawa, K., Moorman, C., Wright, S., \& Christopher, J. (2010). Space-Time Finite Element Computation of Complex Fluid-Structure Interactions. International Journal for Numerical Methods in Fluids, 64, 1201-1218.

[124] Yan, J., Korobenko, A., Tejada-Martinez, A. E., Golshan, R., \& Bazilevs, Y. (2017). A new variational multiscale formulation for stratified incompressible turbulent flows. Computers \& Fluids, 158, 150-156.

[125] van Opstal, T. M., Yan, J., Coley, C., Evans, J. A., Kvamsdal, T., \& Bazilevs, Y. (2017). Isogeometric divergenceconforming variational multiscale formulation of incompressible turbulent flows. Computer Methods in Applied Mechanics and Engineering, 316, 859-879.

[126] Xu, F., Moutsanidis, G., Kamensky, D., Hsu, M.-C., Murugan, M., Ghoshal, A., \& 
Bazilevs, Y. (2017). Compressible flows on moving domains: Stabilized methods, weakly enforced essential boundary conditions, sliding interfaces, and application to gas-turbine modeling. Computers \& Fluids, $158,201-220$.

[127] Hsu, M.-C., Bazilevs, Y., Calo, V. M., Tezduyar, T. E., \& Hughes, T. J. R. (2010). Improving Stability of Stabilized and Multiscale Formulations in Flow Simulations at Small Time Steps. Computer Methods in Applied Mechanics and Engineering, 199, 828-840.

[128] Takizawa, K., Tezduyar, T. E., \& Otoguro, Y. (2018). Stabilization and discontinuity-capturing parameters for space-time flow computations with finite element and isogeometric discretizations. Computational Mechanics, 62, 1169-1186.

[129] Takizawa, K., Ueda, Y., \& Tezduyar, T. E. (2019). A Node-NumberingInvariant Directional Length Scale for Simplex Elements. Mathematical Models and Methods in Applied Sciences, 29, 2719-2753.

[130] Otoguro, Y., Takizawa, K., \& Tezduyar, T. E. (2020). Element Length Calculation in B-Spline meshes for Complex Geometries. Computational Mechanics, published online, DOI: 10.1007/s00466-01901809-w.

[131] Corsini, A., Menichini, C., Rispoli, F., Santoriello, A., \& Tezduyar, T. E. (2009). A Multiscale Finite Element Formulation with Discontinuity Capturing for Turbulence Models with Dominant Reactionlike Terms. Journal of Applied Mechanics, 76, 021211.

[132] Rispoli, F., Saavedra, R., Menichini, F., \& Tezduyar, T. E. (2009). Computation of Inviscid Supersonic Flows around Cylinders and Spheres with the V-SGS Stabilization and YZ $\beta$ Shock-Capturing. Journal of Applied Mechanics, 76, 021209.
[133] Corsini, A., Iossa, C., Rispoli, F., \& Tezduyar, T. E. (2010). A DRD Finite Element Formulation for Computing Turbulent Reacting Flows in Gas Turbine Combustors. Computational Mechanics, 46, 159-167.

[134] Corsini, A., Rispoli, F., \& Tezduyar, T. E. (2011). Stabilized Finite Element Computation of NOx Emission in Aero-engine Combustors. International Journal for Numerical Methods in Fluids, 65, 254-270.

[135] Corsini, A., Rispoli, F., \& Tezduyar, T. E. (2012). Computer modeling of waveenergy air turbines with the SUPG/PSPG formulation and discontinuity-capturing technique. Journal of Applied Mechanics, 79, 010910 .

[136] Corsini, A., Rispoli, F., Sheard, A. G., \& Tezduyar, T. E. (2012). Computational Analysis of Noise Reduction Devices in Axial Fans with Stabilized Finite Element Formulations. Computational Mechanics, $50,695-705$.

[137] Kler, P. A., Dalcin, L. D., Paz, R. R., \& Tezduyar, T. E. (2013). SUPG and discontinuity-capturing methods for coupled fluid mechanics and electrochemical transport problems. Computational Mechanics, 51, 171-185.

[138] Corsini, A., Rispoli, F., Sheard, A. G. Takizawa, K., Tezduyar, T. E., \& Venturini, P. (2014). A variational multiscale method for particle-cloud tracking in turbomachinery flows. Computational Mechanics, 54, 1191-1202.

[139] Rispoli, F., Delibra, G., Venturini, P., Corsini, A., Saavedra, R., \& Tezduyar, T. E. (2015). Particle tracking and particle-shock interaction in compressibleflow computations with the V-SGS stabilization and $\mathrm{YZ} \beta$ shock-capturing. Computational Mechanics, 55, 1201-1209.

[140] Cardillo, L., Corsini, A., Delibra, G. Rispoli, F., \& Tezduyar, T. E. (2016). Flow analysis of a wave-energy air turbine with the SUPG/PSPG stabilization and 
Discontinuity-Capturing Directional Dissipation. Computers \& Fluids, 141, 184190.

[141] Castorrini, A., Corsini, A., Rispoli, F., Venturini, P., Takizawa, K., \& Tezduyar, T. E. (2016). Computational analysis of wind-turbine blade rain erosion. Computers \& Fluids, 141, 175-183.

[142] Castorrini, A., Corsini, A., Rispoli, F., Venturini, P., Takizawa, K., \& Tezduyar, T. E. (2019). Computational analysis of performance deterioration of a wind turbine blade strip subjected to environmental erosion. Computational Mechanics, 64, 1133-1153.

[143] Hughes, T. J. R., Cottrell, J. A., \& Bazilevs, Y. (2005). Isogeometric analysis: CAD, finite elements, NURBS, exact geometry, and mesh refinement. Computer Methods in Applied Mechanics and Engineering, 194, 4135-4195.

[144] Takizawa, K., \& Tezduyar, T. E. (2014). Space-time computation techniques with continuous representation in time (ST-C). Computational Mechanics, 53, 91-99.

[145] Takizawa, K., Takagi, H., Tezduyar, T. E., \& Torii, R. (2014). Estimation of Element-Based Zero-Stress State for Arterial FSI Computations. Computational Mechanics, 54, 895-910.

[146] Takizawa, K., Torii, R., Takagi, H., Tezduyar, T. E., \& Xu, X. Y. (2014). Coronary arterial dynamics computation with medical-image-based time-dependent anatomical models and element-based zero-stress state estimates. Computational Mechanics, 54, 1047-1053.

[147] Takizawa, K., Tezduyar, T. E., \& Sasaki, T. (2018). Estimation of element-based zero-stress state in arterial FSI computations with isogeometric wall discretization. In: Wriggers, P., \& Lenarz, T. (Eds.), Biomedical Technology: Modeling, Experiments and Simulation, Lecture Notes in Applied and Computational Mechanics. Springer, 101-122.
[148] Takizawa, K., Tezduyar, T. E., \& Sasaki, T. (2017). Aorta modeling with the element-based zero-stress state and isogeometric discretization. Computational Mechanics, 59, 265-280.

[149] Sasaki, T., Takizawa, K., \& Tezduyar, T. E. (2019). Aorta Zero-Stress State Modeling with T-Spline Discretization. Computational Mechanics, 63, 13151331.

[150] Sasaki, T., Takizawa, K., \& Tezduyar, T. E. (2019). Medical-Image-Based Aorta Modeling with Zero-Stress-State Estimation. Computational Mechanics, 64, 249271.

[151] Takizawa, K., Tezduyar, T. E., \& Sasaki, T. (2019). Isogeometric hyperelastic shell analysis with out-of-plane deformation mapping. Computational Mechanics, 63, 681-700.

[152] Raknes, S. B., Deng, X., Bazilevs, Y., Benson, D. J., Mathisen, K. M., \& Kvamsdal, T. (2013). Isogeometric rotationfree bending-stabilized cables: Statics, dynamics, bending strips and coupling with shells. Computer Methods in Applied Mechanics and Engineering, 263, 127-143.

[153] Takizawa, K., Spielman, T., \& Tezduyar, T. E. (2011). Space-time FSI modeling and dynamical analysis of spacecraft parachutes and parachute clusters. Computational Mechanics, 48, 345-364.

[154] LIFE TOWER. http:// cosmosunfarm.co.jp/lifetower. html.

[155] Hsu, M.-C., Wang, C., Herrema, A. J., Schillinger, D., Ghoshal, A., \& Bazilevs, Y. (2015). An interactive geometry modeling and parametric design platform for isogeometric analysis. Computers and Mathematics with Applications, 70, 1481-1500.

[156] Resor, B. R. (2013). Definition of a $5 \mathrm{MW} / 61.5 \mathrm{~m}$ Wind Turbine Blade Reference Model. Tech. Rep. SAND20132569, Sandia National Laboratories, Albuquerque, NM. 
[157] Manwell, J. F., McGowan, J. G., \& Rogers, A. L. (2009). Wind Energy Explained: Theory, Design and Application. John Wiley \& Sons.

\section{About Authors}

Yuri BAZILEVS received his Ph.D. from the University of Texas at Austin in 2006, and he is currently the E. Paul Sorensen Professor of Engineering at Brown University. He has been conducting computational fluid mechanics research since 2000, teaching classes on that subject since 2008, and has been conducting computational FSI research since 2005. He has published over 140 Web-of-Science-indexed journal articles on computational fluid and structural mechanics and FSI. He is a Web of Science Highly Cited Researcher. He coauthored a book on isogeometric analysis, a technique widely used in computational mechanics, and FSI. He co-authored a textbook titled Computational Fluid-Structure Interaction: Methods and Applications, Published by Wiley, with the Japanese translation published by Morikita Publishing Company. $\mathrm{He}$ is an Associate Editor of Elsevier journal Computers and Fluids and is responsible for the manuscript on computational fluid mechanics and FSI. More information on Bazilevs can be found at http://vivo.brown.edu/display/ybazilev.

Kenji TAKIZAWA received his Ph.D. from Tokyo Institute of Technology in 2005, and he is currently a Professor in the Department of Modern Mechanical Engineering at Waseda University. He has been conducting computational fluid mechanics research since 2000, teaching classes on that subject since 2010, and has been conducting computational FSI research since 2003. He has published over 90 Web-of-Science-indexed journal articles on computational fluid and structural mechanics and FSI. He is a Web of Science Highly Cited Researcher. He coFluids and is responsible authored a textbook titled Computational Fluid-Structure Interaction: Methods and Applications, Published by Wiley, with the Japanese translation published by Morikita
Publishing Company. He served as an Associate Editor of ASME Journal of Applied Mechanics and was responsible for the manuscripts on computational fluid mechanics and FSI. More information on Takizawa can be found at http://www.jp.tafsm.org/.

Tayfun E. TEZDUYAR received his Ph.D. from Caltech in 1982, and he is currently the James F. Barbour Professor of Mechanical Engineering at Rice University. He has been conducting computational fluid mechanics research since 1979, teaching classes on that subject since 1987, and has been conducting computational FSI research since 1991. He has published over 240 Web-of-Science-indexed journal articles on computational fluid and structural mechanics and FSI. He is a Web of Science Highly Cited Researcher. He coauthored a textbook titled Computational Fluid-Structure Interaction: Methods and Applications, Published by Wiley, with the Japanese translation published by Morikita Publishing Company. $\mathrm{He}$ is an Editor of Springer journal Computational Mechanics and is responsible for the manuscripts on computational fluid mechanics and FSI. More information on Tezduyar can be found at http://www.jp.tafsm.org/tezduyar.

Ming-Chen HSU received his Ph.D. from the University of California, San Diego in 2012, and he is currently an Associate Professor in the Department of Mechanical Engineering at Iowa State University. He has been conducting computational fluid mechanics research since 2006, teaching classes on that subject since 2013, and he has been conducting computational FSI research since 2008. He has published over 60 Web-of-Science-indexed journal articles on computational fluid and structural mechanics and FSI. He is a Web of Science Highly Cited Researcher. More information on Tezduyar can be found at http://web.me.iastate.edu/jmchsu. 